\title{
AN INTEGRODIFFERENTIAL APPROACH TO MODELING, CONTROL, STATE ESTIMATION AND OPTIMIZATION FOR HEAT TRANSFER SYSTEMS
}

\author{
Andreas RAUH ${ }^{a, *}$, LuISE SENKEL $^{a}$, HARALd ASCHEMANN $^{a}, \operatorname{VASILY~V.~SAURIN~}^{b}$, \\ GEORGY V. KOSTIN ${ }^{b, c}$ \\ ${ }^{a}$ Chair of Mechatronics \\ University of Rostock, Justus-von-Liebig-Weg 6, D-18059 Rostock, Germany \\ e-mail: \{Andreas.Rauh, Luise.Senkel, Harald.Aschemann\} @uni-rostock.de
}

${ }^{b}$ Institute for Problems in Mechanics

Russian Academy of Sciences, Pr. Vernadskogo 101-1, 119526, Moscow, Russia e-mail: saurineipmnet.ru

${ }^{c}$ Chair of Mechanics and Control Processes

Moscow Institute of Physics and Technology, Moscow, Russia

e-mail: kostin@ipmnet.ru

\begin{abstract}
In this paper, control-oriented modeling approaches are presented for distributed parameter systems. These systems, which are in the focus of this contribution, are assumed to be described by suitable partial differential equations. They arise naturally during the modeling of dynamic heat transfer processes. The presented approaches aim at developing finitedimensional system descriptions for the design of various open-loop, closed-loop, and optimal control strategies as well as state, disturbance, and parameter estimation techniques. Here, the modeling is based on the method of integrodifferential relations, which can be employed to determine accurate, finite-dimensional sets of state equations by using projection techniques. These lead to a finite element representation of the distributed parameter system. Where applicable, these finite element models are combined with finite volume representations to describe storage variables that are-with good accuracy — homogeneous over sufficiently large space domains. The advantage of this combination is keeping the computational complexity as low as possible. Under these prerequisites, real-time applicable control algorithms are derived and validated via simulation and experiment for a laboratory-scale heat transfer system at the Chair of Mechatronics at the University of Rostock. This benchmark system consists of a metallic rod that is equipped with a finite number of Peltier elements which are used either as distributed control inputs, allowing active cooling and heating, or as spatially distributed disturbance inputs.
\end{abstract}

Keywords: heat transfer, predictive control, optimal control, state and disturbance estimation, distributed parameter systems, sensitivity analysis.

\section{Introduction}

In recent years, the modeling of systems with spatiotemporal dynamics and the design of optimal and adaptive control strategies for such systems have been studied actively. These systems are part of many applications in science and engineering, involving processes such as heat transfer, diffusion, and convection. In the following, a brief overview of related research

\footnotetext{
${ }^{*}$ Corresponding author
}

is given which deals with different methodologies for modeling as well as feedforward, feedback, and optimal control for distributed parameter systems (Rauh et al., 2012b; Saurin et al., 2012).

The theoretical foundation for optimal control problems with linear partial differential equations (PDEs) and convex functionals was established by Lions (1971). In the work of Tao (2003), efficient adaptive control approaches, including model reference adaptive control, adaptive pole placement, and adaptive backstepping, 
were presented and analyzed. The book of Krstic and Smyshlyaev (2010) introduces a comprehensive methodology for adaptive control of parabolic PDEs with unknown functional parameters, including reaction-convection-diffusion systems ubiquitous in chemical, thermal, biomedical, aerospace, and energy systems.

If the derivation of real-time capable control strategies is of interest, two fundamentally different approaches can be distinguished (see Deutscher and Harkort, 2008; 2010). In late lumping procedures, infinite-dimensional control strategies are developed which are approximated by (finite-dimensional) series representations at the latest possible design stages to obtain procedures that have sufficiently low numerical complexity. As modeling and control design are strongly interwoven in these approaches, they are often restricted to special input/output configurations (Kharitonov and Sawodny, 2006; Meurer and Zeitz, 2003; Winkler and Lohmann, 2009; Meurer and Kugi, 2009).

This is due to the fact that special system properties such as differential flatness or linearity assumptions are advantageously exploited in many research articles (Malchow and Sawodny, 2011; Utz et al., 2011; Thull et al., 2010; Touré and Rudolph, 2002; Gehring et al., 2012; Bachmayer et al., 2011). Moreover, these restrictions also involve assumptions on the structure of boundary conditions which are not always fulfilled in practice. Hence, alternative early lumping approaches are often more flexible if a finite-dimensional approximation of models with spatiotemporal dynamics is of interest. This is especially true if real-time applicable control techniques are developed.

Classical early lumping approaches make use of finite volume, finite element or finite difference schemes to reduce the original initial-boundary value problem to a system of ordinary differential equations (ODEs) or-if the model was discretized in both space and time-to systems of algebraic equations. However, the drawback of many classical early lumping techniques is the fact that they do not allow a rigorous quantification of the resulting approximation quality. Therefore, the method of integrodifferential relations (MIDR) has been proposed by the authors to obtain finite-dimensional system models for control purposes with an approximation quality that can be quantified by (energy-related) error measures. For example, in the work of Kostin and Saurin (2006) this method was employed for optimal control design of elastic beam motions, while a variational principle has been applied by Aschemann et al. (2010) on the basis of an MIDR formulation for a parabolic PDE. This latter system describes an application from the field of heat transfer for which accurate trajectory tracking is the main control objective. Moreover, a projection approach, which is also based on the MIDR, has been developed by Rauh et al. (2010) for the same application. Both of these publications are the basis for the experimental case study for a spatially one-dimensional heat transfer process in this paper. For further information concerning the theoretical background of the MIDR, see the work of Kostin and Saurin (2012). Possible extensions of this approach to a problem-oriented modeling of higher-dimensional applications can be found in the works of Rauh et al. (2013b) and Kersten et al. (2014). Additionally, strategies for a order reduction-aiming at real-time applicability of the finite element model in control and state estimation-are described exemplarily by Rauh et al. (2015).

In this paper, a projection formulation of the MIDR is combined with a finite element modeling scheme to describe the space and time dependency of the temperature distribution in rod-like structures; cf. Section 2 These system models are combined with finite volume representations-assuming piecewise homogeneous distributions of the temperature over finitely large domains - to account for disturbances that are caused by convective heat transfer as soon as the ambient temperature is subject to variations. With the help of these models, predictive and optimal control strategies are developed and implemented experimentally for the before-mentioned rod-like distributed heating system. As shown by Saurin et al. (2011a; 2011b), the problem of tracking control can be solved efficiently by combining adaptive control approaches with the MIDR if non-negligible external disturbances or uncertain parameters influence the system behavior. To make the developed control procedures robust against measurement noise and external disturbances, an online applicable state and disturbance observer is described in Section 3 Here, typical disturbances are variations of the ambient temperature and non-modeled external heat flows. This estimation approach is validated experimentally for real-time implementation of an optimal controller (Rauh et al., 2012b). Finally, the observer-based control architecture is extended in Section 4 to the design of a predictive control strategy which has proven its efficiency in cases in which the system becomes non-linear due to the mass flow dependency of coefficients for convective heat transfer between the rod and the ambient medium. Conclusions and an outlook on future research are given in Section 5 .

\section{Application-oriented benchmark for modeling distributed heating systems by the method of integrodifferential relations (MIDR)}

In this paper, modeling procedures with a quantifiable approximation accuracy are developed for distributed 
heating systems. These models are a prerequisite for a reliable control of this kind of systems. The focus of the presented modeling approaches is on spatially one-dimensional scenarios. However, these models can be generalized to higher-dimensional applications (see Rauh et al., 2013b; Kersten et al., 2014). Note that the modeling and control aspects in this paper are selected in such a way that they allow highlighting all fundamental properties of the MIDR procedure.

2.1. Spatially one-dimensional benchmark application. In Fig. 1, the benchmark application used for modeling as well as control and observer design is depicted. It is a distributed heating system consisting of a metallic rod that can be heated or cooled from below by four independent Peltier elements. These Peltier elements with the heat flows $\dot{Q}_{\mathrm{H} i}, i \in\{1, \ldots, 4\}$, either serve as distributed control or disturbance inputs. In addition, an air canal is fixed to the top of the metallic rod which can be used for active cooling. Besides the air mass flow, the temperatures at the geometric midpoints of the four rod segments (each using PT100 resistance sensors) and at the midpoints of the air canal elements 5 and 8 (using thermocouples) are measured.

With this setup, the following two control tasks can be validated in experiments:

1. Tracking of a desired (or optimized) temperature profile in one of the rod segments, where a single Peltier element is used as a controlled heat source (input variable). All other Peltier elements and the time-varying temperature of the air canal are interpreted as a-priori unknown disturbances that have to be compensated efficiently by the controller; see Section 3 .

2. Use of a fan with adjustable speed connected to the air canal as the control input to prevent the violation of an upper bound for the admissible temperature at the position where the maximum rod temperature is expected. In this case, all Peltier elements play the role of disturbance inputs. The predictive control law should reduce the fan speed as far as possible for operating conditions in which the rod temperature falls below its threshold value. Moreover, high frequency variations of the fan speed should be avoided for the bounded air mass flow $\dot{m} \in$ $\left[0 \mathrm{~kg} / \mathrm{s}, \dot{m}_{\text {max }}\right]$; see Section 4 Note that the location of the maximum rod temperature has to be estimated in real time by means of a suitable observer. This observer is based on a suitable coupling of two dynamic models: (i) the model for the temperature distribution in the rod and (ii) the model for the temperature variation in the air canal.

Throughout this paper, the fixed system parameters are given by the length $l=0.32 \mathrm{~m}$ of the rod and air canal (which are each subdivided into $N=4$ segments according to the locations of the Peltier elements), the height $h_{\mathrm{RE}}=0.012 \mathrm{~m}$ of the rod, its width $b_{\mathrm{RE}}=$ $0.040 \mathrm{~m}$, the heat conductivity $\lambda_{\mathrm{R}}=110 \mathrm{~W} /(\mathrm{m} \cdot \mathrm{K})$, the height $h_{\mathrm{AE}}=0.015 \mathrm{~m}$, the convective heat transfer coefficient $\alpha_{\mathrm{R}}=50 \mathrm{~W} /\left(\mathrm{m}^{2} \cdot \mathrm{K}\right)$ between the rod and the air canal for $\dot{m}=0$, the rod density $\rho_{\mathrm{R}}=7800 \mathrm{~kg} / \mathrm{m}^{3}$, its specific heat capacity $c_{\mathrm{R}}=420 \mathrm{~J} /(\mathrm{kg} \cdot \mathrm{K})$, and the corresponding parameters $\rho_{\mathrm{A}}$ and $c_{\mathrm{A}}$ of air.

2.2. Finite volume discretization. As shown in Fig. 11 a basic finite-dimensional model can be derived if the rod and the air canal are discretized into a finite number of segments (Rauh et al., 2012c). If the number of rod segments and the number of Peltier elements $(N=4)$ are chosen equal, heat flow balances between directly neighboring segments lead to a system of ODEs for the rod temperatures,

$$
\begin{aligned}
& \dot{\vartheta}_{1, \mathrm{FV}}=K_{1} \dot{Q}_{\mathrm{H} 1}-\vartheta_{1}^{1,2}-\vartheta_{2}^{1,8}, \\
& \dot{\vartheta}_{2, \mathrm{FV}}=K_{1} \dot{Q}_{\mathrm{H} 2}+\vartheta_{1}^{1,2}-\vartheta_{1}^{2,3}-\vartheta_{2}^{2,7}, \\
& \dot{\vartheta}_{3, \mathrm{FV}}=K_{1} \dot{Q}_{\mathrm{H} 3}+\vartheta_{1}^{2,3}-\vartheta_{1}^{3,4}-\vartheta_{2}^{3,6}, \\
& \dot{\vartheta}_{4, \mathrm{FV}}=K_{1} \dot{Q}_{\mathrm{H} 4}+\vartheta_{1}^{3,4}-\vartheta_{2}^{4,5},
\end{aligned}
$$

and the air canal temperatures,

$$
\begin{aligned}
& \dot{\vartheta}_{5, \mathrm{FV}}=\dot{m} \vartheta_{3}^{6,5}-\vartheta_{4}^{5, \mathrm{~A}}+\vartheta_{5}^{4,5}, \\
& \dot{\vartheta}_{6, \mathrm{FV}}=\dot{m} \vartheta_{3}^{7,6}-\vartheta_{4}^{6, \mathrm{~A}}+\vartheta_{5}^{3,6}, \\
& \dot{\vartheta}_{7, \mathrm{FV}}=\dot{m} \vartheta_{3}^{8,7}-\vartheta_{4}^{7, \mathrm{~A}}+\vartheta_{5}^{2,7}, \\
& \dot{\vartheta}_{8, \mathrm{FV}}=\dot{m} \vartheta_{3}^{\mathrm{I}, 8}-\vartheta_{4}^{8, \mathrm{~A}}+\vartheta_{5}^{1,8}
\end{aligned}
$$

with the parameters

$$
\begin{aligned}
K_{1} & =\frac{1}{\rho_{\mathrm{R}} c_{\mathrm{R}} V_{\mathrm{RE}}}, & p_{1} & =K_{1} \frac{\lambda_{\mathrm{R}} A_{\mathrm{RC}}}{l_{\mathrm{RE}}}, \\
p_{2} & =K_{1} \alpha_{\mathrm{R}} A_{\mathrm{RE}}, & p_{3} & =\frac{1}{\rho_{\mathrm{A}} V_{\mathrm{AE}}}, \\
p_{4} & =\frac{p_{3} \alpha_{\mathrm{A}} A_{\mathrm{AE}}}{c_{\mathrm{A}}}, & p_{5} & =\frac{p_{3} \alpha_{\mathrm{R}} A_{\mathrm{RE}}}{c_{\mathrm{A}}},
\end{aligned}
$$

and $\vartheta_{i}^{j, k}=p_{i} \cdot\left(\vartheta_{j, \mathrm{FV}}-\vartheta_{k, \mathrm{FV}}\right)$. In (1), (2), it is assumed that all rod surfaces that are not in direct contact with the air canal are adiabatically insulated. This information serves as a virtual measurement that further becomes relevant for the observer approaches in Section 3

In the model (1), (2), heat conduction is taken into account in the metallic rod (density $\rho_{\mathrm{R}}$, heat capacity $c_{R}$ ) by the coefficient $\lambda_{R}$. Moreover, convective heat transfer processes between the rod and the air canal, as well as between the air canal and the ambient air (coefficients $\alpha_{\mathrm{R}}$ and $\alpha_{\mathrm{A}}$ ), are included in the ODEs (1) and (2). Furthermore, the transport of air with the density $\rho_{\mathrm{A}}$ and the specific heat capacity $c_{\mathrm{A}}$ in the canal is characterized by the mass flow $\dot{m}$. The finite volume 


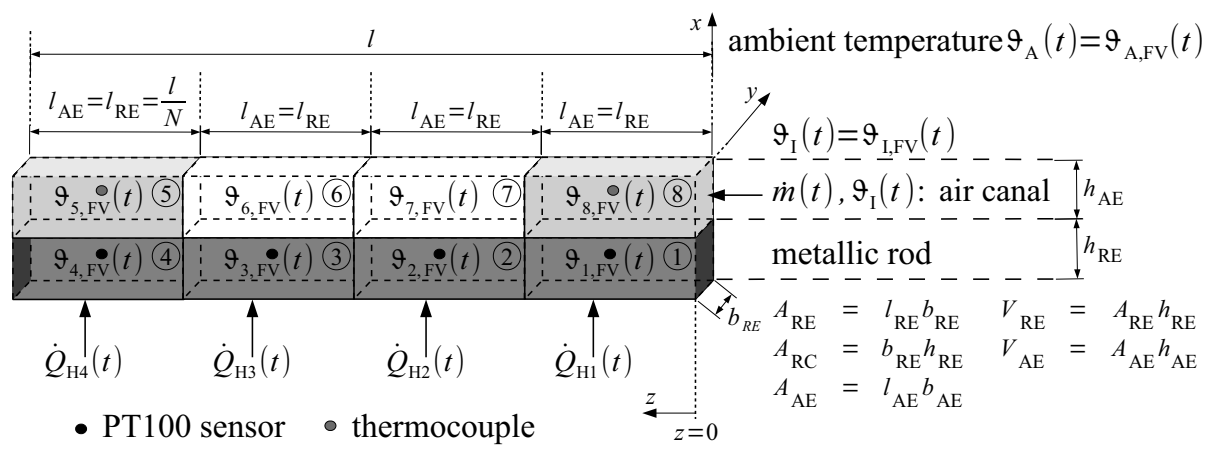

Fig. 1. Experimental setup for the distributed heating system including all sensor locations (RE: rod element, RC: rod cross section, $\mathrm{AE}$ : air canal element, FV: finite volume representation).

approximation (1), (2) accounts for the enthalpy flow of air as a system input in the first terms of the ODEs for the temperatures $\vartheta_{5, \mathrm{FV}}, \ldots, \vartheta_{8, \mathrm{FV}}$ with the air canal inlet temperature $\vartheta_{\mathrm{I}}=\vartheta_{\mathrm{I}, \mathrm{FV}}$, cf. Fig. 1 with a list of all further parameters. Since $l \gg h_{\mathrm{RE}}$ and $l \gg b_{\mathrm{RE}}$, system models treating the position variable $z$ as the only relevant space coordinate are sufficiently accurate for control purposes. These models neglect space dependencies in the $x$ and $y$ coordinates, except for a step-like variation of the temperature between the rod and the air canal.

All Peltier elements serve as distributed heat sources with homogeneous heat flows $\dot{Q}_{\mathrm{H} i}, i \in\{1, \ldots, 4\}$, along the $i$-th rod segment. In the experiments presented in this paper, these heat flows are determined by subsidiary control strategies which make use of a Peltier element model relating the heat flows $\dot{Q}_{\mathrm{H} i}$ to the supply voltages provided by suitable power controllers. According to Rauh and Aschemann (2012), the mass flow dependency of all system parameters $p_{1}=p_{1}(\dot{m}), \ldots$, $p_{5}=p_{5}(\dot{m})$ has been identified experimentally for the case of $\dot{m}(t)>0$.

A drawback of this finite volume model is the rough spatial resolution of the temperature distribution in the metallic rod (leading to piecewise homogeneous values) if the finite volume model is applied. For that reason, the MIDR is introduced in the following subsection to describe the rod temperature more accurately and to allow a model-based detection of the rod position at which the maximum temperature occurs.

2.3. Integrodifferential statement of the onedimensional heat transfer problem. The spatial resolution of the approximation for the temperature distribution in the metallic rod can be improved by employing the MIDR system formulation. In this case, the distributed parameter model for the spatially one-dimensional heat transfer process is split up into a constitutive relation and a corresponding energy balance.

The constitutive relation is the heat flux law (Fourier's law) coupling the heat flux density $q(z, t)$ with the temperature gradient in the interior of the metallic rod according to

$$
\xi(\vartheta(z, t), q(z, t)):=q(z, t)+\lambda_{\mathrm{R}} \frac{\partial \vartheta(z, t)}{\partial z}=0 .
$$

Basically, both the heat flux density $q(z, t)$ and the temperature distribution $\vartheta(z, t)$ are treated as the exact values $q^{*}(z, t)$ and $\vartheta^{*}(z, t)$ that arise in the benchmark application. In contrast to the previous finite volume model, it is assumed-for the derivation of the MIDR - that the air mass flow $\dot{m}=0$ and that the temperature in the air canal is given by the corresponding profile $\vartheta_{\mathrm{AC}}(z, t)$.

The energy balance (first law of thermodynamics) leads to the expression

$$
\frac{\partial q(z, t)}{\partial z}+\kappa_{1} \frac{\partial \vartheta(z, t)}{\partial t}+\kappa_{2} \vartheta(z, t)=\mu(z, t) .
$$

In [5], the parameters $\kappa_{1}=\rho_{\mathrm{R}} c_{\mathrm{R}}$ and $\kappa_{2}=\alpha_{\mathrm{R}} h_{\mathrm{RE}}^{-1}$ characterize the heat capacity and the heat transfer to the air canal, respectively. The function $\mu(z, t), 0 \leq$ $z \leq l$, represents distributed control inputs as well as disturbances along the length of the rod (both provided by the Peltier elements). Moreover, it accounts for variations of the air canal temperature $\vartheta_{\mathrm{AC}}(z, t)$ in space and time.

In such a way, the function $\mu(z, t)$ can be stated as

$$
\mu(z, t)=\mu_{\mathrm{d}}(z, t)+\mu_{\mathrm{c}}(z, t)
$$

with $\mu_{\mathrm{d}}(z, t)=a_{\mathrm{d}} \vartheta_{\mathrm{AC}}(z, t), a_{\mathrm{d}}=\kappa_{2}$, and

$$
\mu_{c}(z, t)=\sum_{i=1}^{N} a_{\mathrm{c}, i}(z) \dot{Q}_{\mathrm{H} i}(t)
$$

where

$$
a_{\mathrm{c}, i}(z)= \begin{cases}\frac{1}{b_{\mathrm{RE}} h_{\mathrm{RE}} l_{\mathrm{RE}}} & \text { for } z_{i-1}<z<z_{i} \\ 0 & \text { otherwise. }\end{cases}
$$

In (8), the positions $z_{0}, \ldots, z_{N}$ denote the edges of the Peltier elements in the space direction $z$. 
In terms of the heat flux density $q(z, t)$, the boundary conditions for the one-dimensional heat transfer process are given by $q(0, t)=\bar{q}_{0}(t)$ and $q(l, t)=\bar{q}_{l}(t)$ at both edges of the rod. In the case of an adiabatic insulation of the rod at both ends $z=0$ and $z=l$ according to Section 2.2 these generally time-dependent boundary conditions simplify to $\bar{q}_{0}(t)=0$ and $\bar{q}_{l}(t)=0$. To make the formulation of the initial-boundary value problem complete, the initial temperature distribution in the rod has to be specified according to

$$
\vartheta(z, 0)=\bar{\vartheta}_{0}(z) .
$$

Without any loss of generality, $\bar{\vartheta}_{0}(z)$ can be set equal to the ambient temperature $\vartheta_{\mathrm{A}}(t=0)=\vartheta_{\mathrm{I}}(t=0)$ in all simulations and experiments (corresponding to an initialization with the thermodynamic equilibrium).

Integrating (5) with respect to the coordinate $z$ and taking into account the boundary condition $q(0, t)=\bar{q}_{0}(t)$ lead to an explicit expression for the heat flux density

$$
\begin{aligned}
q(z, t) & =\bar{q}_{0}(t) \\
& +\int_{0}^{z}\left[\mu(x, t)-\kappa_{1} \frac{\partial \vartheta(x, t)}{\partial t}-\kappa_{2} \vartheta(x, t)\right] \mathrm{d} x .
\end{aligned}
$$

Then, the second boundary condition $q(l, t)=\bar{q}_{l}(t)$ is included in a linear integrodifferential equation according to

$$
\begin{aligned}
\int_{0}^{l}\left[\kappa_{1} \frac{\partial \vartheta(x, t)}{\partial t}+\kappa_{2} \vartheta(x, t)\right] \mathrm{d} x \\
=\int_{0}^{l} \mu(x, t) \mathrm{d} x+\bar{q}_{0}(t)-\bar{q}_{l}(t) .
\end{aligned}
$$

Using the expression (10) for the heat flux density $q(z, t)$, the constitutive relation (4) can be rewritten as

$$
\begin{aligned}
\xi(z, t, \vartheta) & :=\lambda_{\mathrm{R}} \frac{\partial \vartheta(z, t)}{\partial z} \\
& +\int_{0}^{z}\left[\mu(x, t)-\kappa_{1} \frac{\partial \vartheta(x, t)}{\partial t}-\kappa_{2} \vartheta(x, t)\right] \mathrm{d} x \\
& +\bar{q}_{0}(t)=0 .
\end{aligned}
$$

To solve the corresponding initial-boundary value problem (9), (11), (12), the MIDR is applied in which the constitutive relation (12) is replaced according to Rauh et al. $(2012 \mathrm{~b})$ by the integral relation

$$
\Phi(\vartheta)=\int_{0}^{t_{\mathrm{f}}} \int_{0}^{l} \varphi(z, t, \vartheta) \mathrm{d} z \mathrm{~d} t=0
$$

with $\varphi(z, t, \vartheta):=\xi^{2}(z, t, \vartheta)$. In (13), the interval $\left[0, t_{\mathrm{f}}\right]$ denotes the time horizon over which the process is considered with the given terminal instant $t_{\mathrm{f}}$.

Thus, the initial-boundary value problem can be reformulated: Find a temperature distribution $\vartheta^{*}(z, t)$ that obeys the initial condition $\vartheta(z, 0)=\bar{\vartheta}_{0}(z)$ according to (9) as well as the boundary conditions $\bar{q}_{0}(t)$ and $\bar{q}_{l}(t)$ and simultaneously satisfies the integral relation (13).

Since it is not always possible to solve the integrodifferential formulation of the boundary value problem exactly, approximations $\tilde{\vartheta}(z, t)$ to the true temperature distribution $\vartheta(z, t)=\vartheta^{*}(z, t)$ are determined in the following. In this case, the integrodifferential formulation provides the possibility to estimate the quality of $\tilde{\vartheta}(z, t)$.

As the integrand $\varphi(z, t, \vartheta)$ in (13) is guaranteed to be non-negative, the integral $\tilde{\Phi}=\Phi(\tilde{\vartheta})$ is always non-negative and reaches its absolute minimum $\Phi=$ 0 solely on the exact solution $\vartheta(z, t) \equiv \vartheta^{*}(z, t)$ (see Aschemann et al., 2010). This holds for any admissible temperature distribution $\tilde{\vartheta}(z, t)$ satisfying the initial conditions, boundary conditions as well as the energy conservation law. Therefore, the value $\tilde{\Phi}=\Phi(\tilde{\vartheta}) \neq 0$ defined according to (13) serves as a measure for the integral quality of the approximate solution $\tilde{\vartheta}(z, t)$. Additionally, the integrand $\varphi(z, t, \tilde{\vartheta})$ shows the local error distribution in both space and time.

Note that the dimensionless ratio

$$
\Delta=\frac{\tilde{\Phi}}{\tilde{\Psi}}, \quad \tilde{\Psi}=\int_{0}^{t_{\mathrm{f}}} \int_{0}^{l}\left(\lambda_{\mathrm{R}} \frac{\partial \tilde{\vartheta}(z, t)}{\partial z}\right)^{2} \mathrm{~d} z \mathrm{~d} t
$$

can be used as the relative integral error of any admissible temperature field. In (14), the dimensionless error measure is defined as the ratio between the integral square error in the approximation of the heat flux density and the corresponding square value of this approximation. A suitable approximation $\tilde{\vartheta}(z, t)$ can be determined by either directly minimizing the term $\Delta$ or by minimizing the corresponding numerator $\tilde{\Phi}$. This leads to the variational problem formulation given by Saurin et al. (2011b). Leaving out the time integral in (13) corresponds to the optimization-based solution described by Rauh et al. (2012b). Alternatively, the following projection scheme can be employed. For all three options (namely, the projection, variational, and optimization-based formulations), combinations with a finite element discretization of the temperature field are reasonable to keep the approximations $\tilde{\vartheta}(z, t)$ as simple as possible. Here, it is typically desired to find polynomial approximations for the temperature distribution with the smallest possible degree.

\subsection{Projection approach for finite element mode-} ling. To determine the approximate solution $\tilde{\vartheta}(z, t)$ by solving a set of ODEs that corresponds to a projection formulation of the aforementioned integrodifferential problem statement, it is assumed that the temperature in the spatially one-dimensional heat transfer problem is described by a piecewise polynomial approximation of the 
unknown temperature distribution $\vartheta^{*}(z, t)$, cf. (Rauh et al., 2012b).

For that purpose, the rod length $z \in[0, l]$ is divided into $N$ finite elements with $z \in\left[z_{i-1}, z_{i}\right], i=1, \ldots, N$, where $0=z_{0}<z_{1}<\ldots<z_{N-1}<z_{N}=l$ are the nodal coordinates. For the sake of simplicity, it is assumed that the number of Peltier elements in Fig. 2 is equal to the number $N$ of finite elements.

Hence, the approximation $\tilde{\vartheta}(z, t)$ of the temperature profile is defined by

$$
\begin{aligned}
& \tilde{\vartheta}(z, t)=\sum_{i=1}^{N} \sum_{k=0}^{M} b_{i, k, M}(z) \cdot \theta_{i, k, M}(t), \\
& \tilde{\vartheta}(z, t)=\vartheta_{i, \mathrm{FE}}(z, t) \quad \text { for } \quad z \in\left[z_{i-1}, z_{i}\right],
\end{aligned}
$$

where $\theta_{i, k, M}(t)$ are unknown time-dependent coefficients and $M$ is the fixed polynomial degree of the functions $b_{i, k, M}(z)$ with respect to the coordinate $z$. For numerical reasons and to simplify the computation of the required inter-element conditions, Bernstein polynomials

$$
b_{i, k, M}(z)= \begin{cases}b_{i}^{k, M}(z) & \text { for } z \in\left[z_{i-1}, z_{i}\right] \\ 0 & \text { otherwise }\end{cases}
$$

of degree $M$ are used instead of pure monomials $z^{k}$ to approximate the temperature distribution in each rod segment, where

$$
b_{i}^{k, M}(z)=\left(\begin{array}{c}
M \\
k
\end{array}\right)\left(\frac{z-z_{i-1}}{z_{i}-z_{i-1}}\right)^{k}\left(\frac{z_{i}-z}{z_{i}-z_{i-1}}\right)^{M-k} .
$$

The continuity of the temperature distribution at the common node $z_{i}$ between two directly neighboring finite elements $i$ and $i+1$ is guaranteed by the relation

$$
\theta_{i, M, M}(t)=\theta_{i+1,0, M}(t) .
$$

To simplify the notation, vectors

$$
\boldsymbol{b}_{i, M}(z)=\left[\begin{array}{lll}
b_{i, 0, M}(z) & \ldots & b_{i, M, M}(z)
\end{array}\right]^{T}
$$

and

$$
\boldsymbol{B}_{M}(z)=\left[\begin{array}{lll}
\boldsymbol{b}_{1, M}^{T}(z) & \ldots & \boldsymbol{b}_{N, M}^{T}(z)
\end{array}\right]^{T}
$$

are introduced to denote all Bernstein polynomials of order $M$ for either one rod segment $i$ or for the union of all segments, respectively 1

Accordingly, the coefficient vectors

$$
\boldsymbol{\theta}_{i, M}(t)=\left[\begin{array}{lll}
\theta_{i, 0, M}(t) & \ldots & \theta_{i, M, M}(t)
\end{array}\right]^{T}
$$

and

$$
\boldsymbol{\Theta}_{M}(t)=\left[\begin{array}{lll}
\boldsymbol{\theta}_{1, M}^{T}(t) & \ldots & \boldsymbol{\theta}_{N, M}^{T}(t)
\end{array}\right]^{T}
$$

\footnotetext{
${ }^{1}$ To make the short-hand notation in 15 and 21 non-ambiguous, $b_{i, k, M}\left(z_{i}\right)=0$ is assumed for $i \in\{1, \ldots, N-1\}$.
}

are defined. Hence, $\tilde{\vartheta}(z, t)$ in (15) can be replaced with

$$
\tilde{\vartheta}(z, t)=\sum_{i=1}^{N} \boldsymbol{\theta}_{i, M}^{T}(t) \boldsymbol{b}_{i, M}(z)=\boldsymbol{\Theta}_{M}^{T}(t) \boldsymbol{B}_{M}(z) .
$$

To determine the set of ODEs for the coefficient vector $\boldsymbol{\Theta}_{M}:=\boldsymbol{\Theta}_{M}(t)$, Bernstein polynomials of order $M-1$ are used as test functions in the following projection approach that replaces the equality (13).

The formulation of a projection relation

$$
\int_{z_{i-1}}^{z_{i}}\left(\xi(z, t, \tilde{\vartheta}) \cdot b_{i, k, M-1}(z)\right) \mathrm{d} z=0
$$

for each finite element $i \in\{1, \ldots, N\}$ as well as for each polynomial degree $k \in\{0, \ldots, M-1\}$ leads to a system of $M \cdot N$ ODEs for the unknown coefficients $\boldsymbol{\Theta}_{M}$. However, after elimination of the coefficients $\theta_{2,0, M}(t), \ldots, \theta_{N, 0, M}(t)$ from the vector $\boldsymbol{\Theta}_{M}$ according to the inter-element conditions (18), there are $M \cdot N+1$ remaining unknowns. The missing relation that has to be appended to the before-mentioned system of ODEs results from the boundary condition with

$$
\begin{gathered}
\int_{0}^{l}\left[\kappa_{1} \frac{\partial \tilde{\vartheta}\left(x, \boldsymbol{\Theta}_{M}\right)}{\partial t}+\kappa_{2} \tilde{\vartheta}\left(x, \boldsymbol{\Theta}_{M}\right)\right] \mathrm{d} x \\
=\int_{0}^{l} \mu(x, t) \mathrm{d} x+\bar{q}_{0}(t)-\bar{q}_{l}(t),
\end{gathered}
$$

where the function $\mu(z, t)$ is defined as given in (6).

Appropriate initial conditions to this set of ODEs are computed from a least-squares approximation of the initial temperature distribution (9) according to

$$
\boldsymbol{\Theta}_{M}^{*}(0)=\arg \min _{\boldsymbol{\Theta}_{M}(0)} \int_{0}^{l}\left(\boldsymbol{\Theta}_{M}^{T}(0) \boldsymbol{B}_{M}(z)-\bar{\vartheta}_{0}(z)\right)^{2} \mathrm{~d} z .
$$

Since Eqns. (22) and (23) are linear in $\boldsymbol{\Theta}_{M}$ and $\dot{\boldsymbol{\Theta}}_{M}$, an explicit set of ODEs can be obtained easily by means of symbolic formula manipulation after eliminating the redundant coefficients $(18)$. The resulting set of ODE 2

$$
\dot{\boldsymbol{x}}(t)=\boldsymbol{A} \boldsymbol{x}(t)+\boldsymbol{B} \boldsymbol{u}(t)+\boldsymbol{E} \boldsymbol{z}(t),
$$

where $\boldsymbol{x}$ includes the non-redundant Bernstein polynomial coefficients $\Theta_{M}$ for the approximation of the temperature distribution. Moreover, the heat flows of all Peltier elements serving as control inputs $\boldsymbol{u}(t)$ and the vector $\boldsymbol{z}(t)$ of all disturbance heat flows provided by the remaining Peltier elements are included as well as the (Bernstein

\footnotetext{
${ }^{2}$ For a symbolic formula manipulation routine, allowing the extraction of the corresponding matrix entries, the reader is referred to the work of Rauh et al. (2013b). Generally, $\operatorname{dim}\{\boldsymbol{x}\}$ is equal to the sum of $M \cdot N+1$ and the number of state variables for the air canal (i.e., $N$ additional state variables $\vartheta_{N+1, \mathrm{FV}}, \ldots, \vartheta_{2 N, \mathrm{FV}}$ for the basic finite volume model).
} 


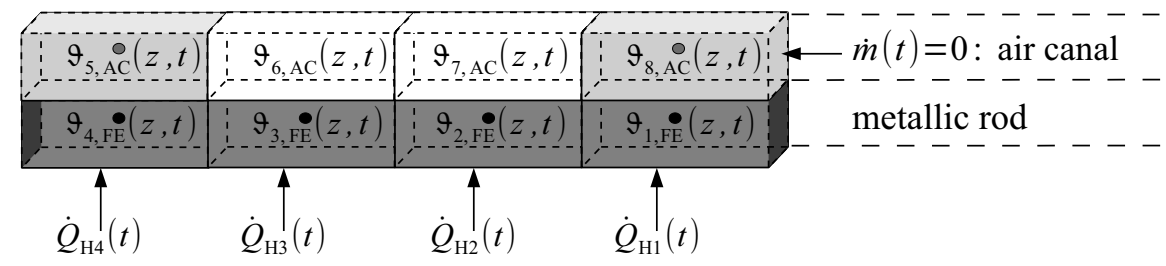

Fig. 2. Finite element representation of the temperature in the heating system (FE: finite element approximation, AC: air canal).

polynomial) representation of the air canal temperature $\vartheta_{\mathrm{AC}}(z, t)$. Assuming that the air canal temperature can be approximated by a piecewise homogeneous distribution, the finite volume element temperatures $\vartheta_{\mathrm{FV}, 5}, \ldots, \vartheta_{\mathrm{FV}, 8}$ can be coupled directly with the above-mentioned finite element representation for the rod temperature; see Fig. 3 and Sections 3 and 4 .

An efficient alternative to this projection approach is the optimization-based solution procedure that has been described in detail by Rauh et al. (2012b). It is characterized by the use of independent ansatz functions for both the temperature distribution and the heat flux density. In such a way, it provides an improved capability of computing accurate approximations to the heat transfer equation. However, this improved accuracy goes along with an increased system dimension. Hence, we restrict ourselves to the previously presented approach for an application of the system model in a real-time control environment. Details on a comparison of the approximation quality of both the approaches can be found in the work of Rauh et al. (2012b). Note that the MIDR can furthermore be employed to quantify the approximation quality of other solution approaches. An example where a finite-dimensional realization of an infinite-dimensional flatness-based control design (implemented as a truncated series expansion) was analyzed is given by Rauh et al. (2010).

\section{Optimal control and model-based observer design}

3.1. Design of optimal feedforward control strategies. Assume that the linear state equations resulting from the projection approach with a fixed degree $M$ and the air mass flow $\dot{m}=0$ are abbreviated by the linear time-invariant state-space representation (25) introduced in Section 2.4 .

The goal of the following control and observer design is the offline computation of an optimal heating strategy and its experimental implementation on the available test rig. In the experiment, the offline computed control input and the corresponding output trajectory are used as a feedforward control sequence and as a reference trajectory, respectively.

To compensate disturbances, the online implementation extends the offline computed feedforward control by a state and output feedback which makes use of estimates for the non-measured components of the vector $\Theta_{M}$ and the external disturbances $\boldsymbol{z}$. This disturbance vector is defined as

$$
\boldsymbol{z}(t)=\left[\begin{array}{llll}
\vartheta_{5, \mathrm{FV}}(t) & \vartheta_{6, \mathrm{FV}}(t) & \vartheta_{7, \mathrm{FV}}(t) & \vartheta_{8, \mathrm{FV}}(t)
\end{array}\right]^{T}
$$

to account for deviations of the temperatures $\vartheta_{5, \mathrm{FV}}(t), \ldots, \vartheta_{8, \mathrm{FV}}(t)$ from the value $\vartheta_{\mathrm{A}} \equiv \vartheta_{5, \mathrm{FV}}(0)=$ $\ldots=\vartheta_{8, \mathrm{FV}}(0)$ that is assumed during the offline optimization of the feedforward control signal. Since these temperature variations are significantly slower than the dynamics of the rod temperature, they are included as an integrator disturbance model with the ODEs

$$
\dot{\boldsymbol{z}}(t)=\left[\begin{array}{llll}
0 & 0 & 0 & 0
\end{array}\right]^{T}
$$

in the observer-based feedback control design that is presented in the following subsections.

Moreover, the control synthesis makes use of the input vector

$$
\begin{aligned}
\boldsymbol{u}(t) & =\left[\begin{array}{llll}
\dot{Q}_{\mathrm{H} 1}(t) & \dot{Q}_{\mathrm{H} 2}(t) & \dot{Q}_{\mathrm{H} 3}(t) & \dot{Q}_{\mathrm{H} 4}(t)
\end{array}\right]^{T} \\
& =\left[\begin{array}{llll}
u(t) & 0 & 0 & 0
\end{array}\right]^{T},
\end{aligned}
$$

so that only the first Peltier element acts as an active heat source and all others are deactivated. In this case, changes in $\boldsymbol{z}(t)$ do not only represent variations of the air temperature above the rod but they also serve as a lumped disturbance variable for effects that are caused by parasitic heat flows (non-ideal adiabatic insulation) through the non-actuated Peltier elements.

3.2. Energy-optimal heating strategy. The goal of the optimal feedforward control synthesis is to transfer the temperature $\tilde{\vartheta}\left(z_{\mathrm{d}}, t\right)$ at a given position $z_{\mathrm{d}}$ in the pre-defined time $t_{\mathrm{f}}$ to a desired final value $\vartheta_{\mathrm{d}}$ with a vanishing final variation rate $\frac{\mathrm{d}}{\mathrm{d} t} \tilde{\vartheta}\left(z_{\mathrm{d}}, t_{\mathrm{f}}\right)=0$.

In the performance criterion

$$
J_{\mathrm{C}}:=f_{\mathrm{T}}+\int_{0}^{t_{\mathrm{f}}} f_{0}(t) \mathrm{d} t \stackrel{!}{=} \min , \quad f_{0}(t)=u^{2}(t),
$$

this goal is taken into account by sufficiently large weighting factors $\nu_{1}$ and $\nu_{2}$ in the terminal cost function

$$
f_{\mathrm{T}}:=\nu_{1} \cdot\left(\tilde{\vartheta}\left(z_{\mathrm{d}}, t_{\mathrm{f}}\right)-\vartheta_{\mathrm{d}}\right)^{2}+\nu_{2} \cdot\left(\frac{\mathrm{d}}{\mathrm{d} t} \tilde{\vartheta}\left(z_{\mathrm{d}}, t_{\mathrm{f}}\right)\right)^{2} .
$$




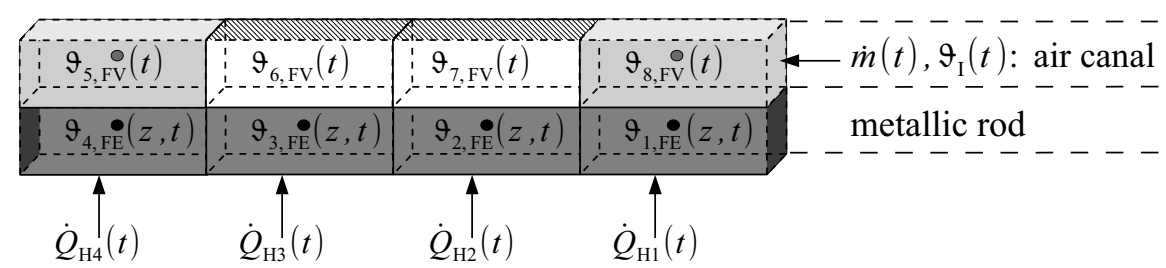

Fig. 3. Coupling of the finite element representation of the rod temperature with a finite volume model for the air canal (with the mass flow-dependent parameters $p_{1}(\dot{m}), \ldots, p_{5}(\dot{m})$ in (1) and (2)).

The minimization of $J_{\mathrm{C}}$ is performed numerically with the help of Pontryagin's maximum principle. For that purpose, the Hamiltonian

$$
\begin{aligned}
H(u(t)):= & -f_{0}(t)+\boldsymbol{p}^{T}(t)(\boldsymbol{A} \boldsymbol{x}(t) \\
& \left.+\boldsymbol{B}[u(t) \quad 0 \quad 0 \quad 0]^{T}+\boldsymbol{E} \boldsymbol{z}(0)\right)
\end{aligned}
$$

with the adjoint states $\boldsymbol{p}=\boldsymbol{p}(t)$ is maximized by the control $u(t)=u_{\text {opt }}(t)$ fulfilling the condition

$$
\left.\frac{\partial H}{\partial u}\right|_{u=u_{\mathrm{opt}}}=0 .
$$

Defining $H_{\mathrm{opt}}:=H\left(u_{\mathrm{opt}}(t)\right)$, the set of canonical equations

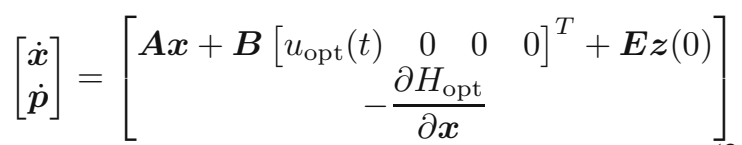

is obtained. The boundary value problem for the Eqns. (32) with the initial states $\boldsymbol{x}(0)=$ $\left[\begin{array}{lll}\vartheta_{\mathrm{A}}(0) & \ldots & \vartheta_{\mathrm{A}}(0)\end{array}\right]^{T}$ and the terminal conditions

$$
\boldsymbol{p}\left(t_{\mathrm{f}}\right)=-\left.\frac{\partial f_{\mathrm{T}}}{\partial \boldsymbol{x}}\right|_{\boldsymbol{x}=\boldsymbol{x}\left(t_{\mathrm{f}}\right)}
$$

is solved numerically by the boundary value problem solver bvp4c in MATLAB. To improve the numerical convergence properties of this solver, an intermediate solution is firstly determined for $\nu_{1}=10^{5}$ and $\nu_{2}=0$. Secondly, this solution is used to re-initialize bvp $4 \mathrm{C}$ with $\nu_{1}=\nu_{2}=10^{5}$.

For the online application, the control input is defined as

$$
u=u_{\mathrm{opt}}+u_{\mathrm{PI}}-\boldsymbol{k}^{T}\left[\begin{array}{c}
\boldsymbol{\theta}_{1, M}-\boldsymbol{I} \vartheta_{8, \mathrm{FV}} \\
\vdots \\
\boldsymbol{\theta}_{4, M}-\boldsymbol{I} \vartheta_{5, \mathrm{FV}}
\end{array}\right],
$$

where $\boldsymbol{I}$ is an identity matrix of appropriate dimensions. All non-measured values $\boldsymbol{\theta}_{i, M}(t), i \in\{1, \ldots, 4\}$, are replaced by the observer outputs described in the following subsection. The control part $u_{\mathrm{PI}}(t)$ represents an additional $\mathrm{P} 3$ (proportional, integrating) output feedback determined by the transfer function

$$
\frac{U_{\mathrm{PI}}(s)}{Y_{\mathrm{d}}(s)-\tilde{\Theta}\left(\frac{7 l}{8}, s\right)}=\left(1+K_{\mathrm{R}} \frac{T_{\mathrm{I}} s+1}{T_{\mathrm{I}} s}\right) S_{\mathrm{v}},
$$

where $s$ is the complex Laplace variable. The feedback and prefilter gains $k$ and $S_{\mathrm{v}}$, respectively, are chosen by a linear quadratic regulator design exploiting the condition for steady-state accuracy. Moreover, $T_{\mathrm{I}}$ compensates the largest time constant of the approximating system model (25) with a fixed value $K_{\mathrm{R}}=3$.

3.3. State and disturbance observer design. Since the vector $\Theta_{M}$ (and therefore also the state vector $\boldsymbol{x}$ ) of the MIDR-based finite element representation is not directly measurable, these values have to be reconstructed during experiments by means of a state observer. This observer is designed in such a way that, furthermore, it reconstructs the disturbance values $z$ defined in 26.

For that purpose, the ODEs (25) obtained from the projection approach in the MIDR are extended by the integrator disturbance models for $z$ according to

$$
\dot{\tilde{\boldsymbol{x}}}(t)=\tilde{\boldsymbol{A}} \tilde{\boldsymbol{x}}(t)+\tilde{\boldsymbol{B}}[u(t) \quad 0 \quad 0 \quad 0]^{T},
$$

where the extended state vector as well as the modified system and input matrices are given by

$$
\tilde{\boldsymbol{x}}:=\left[\begin{array}{c}
\boldsymbol{x} \\
\boldsymbol{z}
\end{array}\right], \quad \tilde{\boldsymbol{A}}:=\left[\begin{array}{cc}
\boldsymbol{A} & \boldsymbol{E} \\
\mathbf{0} & \mathbf{0}
\end{array}\right], \quad \tilde{\boldsymbol{B}}:=\left[\begin{array}{c}
\boldsymbol{B} \\
\mathbf{0}
\end{array}\right] .
$$

Estimates $\hat{\tilde{\boldsymbol{x}}}$ for the non-measurable state vector $\tilde{\boldsymbol{x}}$ are then determined numerically by integrating the differential equations for the linear Luenberger observer

$$
\dot{\hat{\tilde{\boldsymbol{x}}}}=\tilde{\boldsymbol{A}} \hat{\tilde{\boldsymbol{x}}}+\tilde{\boldsymbol{B}}\left[\begin{array}{llll}
u & 0 & 0 & 0
\end{array}\right]^{T}+\boldsymbol{L}(\boldsymbol{y}-\hat{\boldsymbol{y}}),
$$

in which the gain matrix $\boldsymbol{L}$ has to be defined in such a manner that the estimation error dynamics

\footnotetext{
${ }^{3}$ The integral part is included in the control law in order to guarantee steady-state accuracy also in cases in which the non-measured ambient temperature changes. In such a way, the integral feedback helps to compensate non-modeled disturbance heat flows. This equally holds for compensating non-ideal insulation properties at the rod edges and slightly imperfect behavior of the subsidiary heat flow control of the Peltier elements.
} 
becomes asymptotically stable. This can be achieved by a minimization of the integral quadratic error measure

$$
J_{\mathrm{O}}=\frac{1}{2} \int_{0}^{\infty}\left(\Delta \tilde{\boldsymbol{x}}^{T} \boldsymbol{Q} \Delta \tilde{\boldsymbol{x}}+\Delta \boldsymbol{y}^{T} \boldsymbol{R} \Delta \boldsymbol{y}\right) \mathrm{d} t
$$

with weighting matrices $\boldsymbol{Q}=\boldsymbol{Q}^{T} \geq 0$ and $\boldsymbol{R}=$ $\boldsymbol{R}^{T}>0$. Solving this optimization problem with the estimation errors $\Delta \tilde{\boldsymbol{x}}(t)$ (deviations between the true and estimated state vectors) concerning the extended state vector and $\Delta \boldsymbol{y}(t)$ (the difference between the measured and estimated outputs) for the system outputs $\boldsymbol{y}=\boldsymbol{C} \boldsymbol{x}$ leads to the algebraic Riccati equation

$$
\boldsymbol{P} \boldsymbol{C}^{T} \boldsymbol{R}^{-1} \boldsymbol{C P}-\tilde{\boldsymbol{A}} \boldsymbol{P}-\boldsymbol{P} \tilde{\boldsymbol{A}}^{T}-\boldsymbol{Q}=\mathbf{0},
$$

for which a positive definite, symmetric matrix $\boldsymbol{P}=$ $\boldsymbol{P}^{T}>0$ has to be determined (Rauh et al., 2013a; Åström, 1970; Stengel, 1994). Using this matrix $\boldsymbol{P}$, the observer gain is given by

$$
\boldsymbol{L}=\boldsymbol{P} \boldsymbol{C}^{T} \boldsymbol{R}^{-1} .
$$

As shown by Saurin et al. (2012), the matrices $Q$ and $\boldsymbol{R}$ can be set to identity matrices of appropriate dimensions to obtain sufficiently accurate estimation results in simulations and experiments 4 Reasonable definitions for the vectors of system outputs are either

$$
\begin{aligned}
\boldsymbol{y} & =\left[\vartheta_{1, \mathrm{FE}}\left(\frac{l}{8}, t\right), \vartheta_{4, \mathrm{FE}}\left(\frac{7 l}{8}, t\right), \vartheta_{1, \mathrm{FE}}^{\prime}(0, t), \vartheta_{4, \mathrm{FE}}^{\prime}(l, t)\right]^{T} \\
& =\boldsymbol{C}_{1}\left[\begin{array}{c}
\boldsymbol{\Theta}_{M} \\
\boldsymbol{z}
\end{array}\right], \quad \boldsymbol{C}:=\boldsymbol{C}_{1}
\end{aligned}
$$

with

$$
\boldsymbol{C}_{1}=\left[\begin{array}{ccccc}
\boldsymbol{b}_{1, M}^{T}\left(\frac{l}{8}\right) & \mathbf{0}_{M+1}^{T} & \mathbf{0}_{M+1}^{T} & \mathbf{0}_{M+1}^{T} & \mathbf{0}_{4}^{T} \\
\mathbf{0}_{M+1}^{T} & \mathbf{0}_{M+1}^{T} & \mathbf{0}_{M+1}^{T} & \boldsymbol{b}_{4, M}^{T}\left(\frac{7 l}{8}\right) & \mathbf{0}_{4}^{T} \\
\boldsymbol{b}_{1, M}^{\prime T}(0) & \mathbf{0}_{M+1}^{T} & \mathbf{0}_{M+1}^{T} & \mathbf{0}_{M+1}^{T} & \mathbf{0}_{4}^{T} \\
\mathbf{0}_{M+1}^{T} & \mathbf{0}_{M+1}^{T} & \mathbf{0}_{M+1}^{T} & \boldsymbol{b}_{4, M}^{T}(l) & \mathbf{0}_{4}^{T}
\end{array}\right]
$$

or

$$
\begin{aligned}
\boldsymbol{y}= & {\left[\vartheta_{1, \mathrm{FE}}\left(\frac{l}{8}, t\right), \vartheta_{2, \mathrm{FE}}\left(\frac{3 l}{8}, t\right), \vartheta_{3, \mathrm{FE}}\left(\frac{5 l}{8}, t\right),\right.} \\
& \left.\vartheta_{4, \mathrm{FE}}\left(\frac{7 l}{8}, t\right), \vartheta_{1, \mathrm{FE}}^{\prime}(0, t), \vartheta_{4, \mathrm{FE}}^{\prime}(l, t)\right]^{T} \\
= & \boldsymbol{C}_{2}\left[\begin{array}{c}
\boldsymbol{\Theta}_{M} \\
\boldsymbol{z}
\end{array}\right], \quad \boldsymbol{C}:=\boldsymbol{C}_{2}
\end{aligned}
$$

with

$$
\boldsymbol{C}_{2}=\left[\begin{array}{ccccc}
\boldsymbol{b}_{1, M}^{T}\left(\frac{l}{8}\right) & \mathbf{0}_{M+1}^{T} & \mathbf{0}_{M+1}^{T} & \mathbf{0}_{M+1}^{T} & \mathbf{0}_{4}^{T} \\
\mathbf{0}_{M+1}^{T} & \boldsymbol{b}_{2, M}^{T}\left(\frac{3 l}{8}\right) & \mathbf{0}_{M+1}^{T} & \mathbf{0}_{M+1}^{T} & \mathbf{0}_{4}^{T} \\
\mathbf{0}_{M+1}^{T} & \mathbf{0}_{M+1}^{T} & \boldsymbol{b}_{3, M}^{T}\left(\frac{5 l}{8}\right) & \mathbf{0}_{M+1}^{T} & \mathbf{0}_{4}^{T} \\
\mathbf{0}_{M+1}^{T} & \mathbf{0}_{M+1}^{T} & \mathbf{0}_{M+1}^{T} & \boldsymbol{b}_{4, M}^{T}\left(\frac{7 l}{8}\right) & \mathbf{0}_{4}^{T} \\
\boldsymbol{b}_{1, M}^{\prime T}(0) & \mathbf{0}_{M+1}^{T} & \mathbf{0}_{M+1}^{T} & \mathbf{0}_{M+1}^{T} & \mathbf{0}_{4}^{T} \\
\mathbf{0}_{M+1}^{T} & \mathbf{0}_{M+1}^{T} & \mathbf{0}_{M+1}^{T} & \boldsymbol{b}_{4, M}^{T}(l) & \mathbf{0}_{4}^{T}
\end{array}\right],
$$

\footnotetext{
${ }^{4}$ The identical weighing of all components of $\Delta \tilde{\boldsymbol{x}}$ and $\Delta \boldsymbol{y}$ is reasonable since all temperature components as well as the included spatial derivatives of the temperature profile have nominal values of a similar order of magnitude.
}

where zero blocks of appropriate dimensions $\mathbf{0}_{\xi}:=$ $\left[\begin{array}{lll}0 & \ldots & 0\end{array}\right]^{T} \in \mathbb{R}^{\xi}$ are included in $\boldsymbol{C}_{1}$ and $\boldsymbol{C}_{2}$. Note that, according to Fig. 1, both the vectors (42) and (44) contain only values that are (virtually) measurable. In the output corresponding to $C_{1}$, temperature measurements (PT100 sensors) are performed at the midpoints of the first and the last rod segment. In addition, information about adiabatic insulation of both rod edges is taken into account by

$$
\begin{aligned}
\vartheta_{j, \mathrm{FE}}^{\prime}\left(z_{j}^{\prime}, t\right):=\left.\frac{\partial \vartheta_{j, \mathrm{FE}}(z, t)}{\partial z}\right|_{z=z_{j}^{\prime}} & =0, \\
j \in\{1,4\}, \quad z_{j}^{\prime} & \in\left\{z_{0}, z_{N}\right\},
\end{aligned}
$$

and

$$
\boldsymbol{b}_{i, M}^{\prime}(z):=\frac{\mathrm{d} \boldsymbol{b}_{i, M}(z)}{\mathrm{d} z} \quad \text { (defined element wise) }
$$

As shown by the following simulation results, the additional measurements of the temperature values at the midpoints of the second and the third rod element (the output definition using $\boldsymbol{C}_{2}$ ) leads to a further improvement of the observer accuracy5. For all simulations and experiments, the approximation order $M=3$ is chosen. For a detailed justification of this parameter choice, refer to the information about the absolute measure for the approximation error reported by Rauh et al. (2012b).

Figures 4 and 5 show the results for the optimal open-loop control synthesis as well as numerical validation of the quality of the state and disturbance observer with the output definition (42). In Fig. 5, it can be seen that - despite the large initial estimation errors-all rod temperatures (expressed by the coefficients $\boldsymbol{\Theta}_{M}(t)$ ) are estimated accurately after significantly less than $200 \mathrm{~s}$. The swing-in phase for the disturbance vector is longer by a factor of approximately five. However, as shown in the following experimental results, this is sufficient for practical purposes since this duration does not severely influence the control quality.

According to Fig. 6(a) the experimental implementation of the open-loop control, extended by the combined state and output feedback, leads to an accurate tracking of the energy-optimal output trajectory determined by the previously summarized approach. This can be achieved by the disturbance estimation shown in Fig. 6(b) (left). This disturbance has to be counter-acted by the output feedback since it influences

\footnotetext{
${ }^{5}$ Note that the observability of the pairs $\left(\tilde{\boldsymbol{A}} ; \boldsymbol{C}_{1}\right)$ and $\left(\tilde{\boldsymbol{A}} ; \boldsymbol{C}_{2}\right)$ is a fundamental prerequisite for the presented observer approaches. Observability has been checked for the polynomial orders $M=3$ and $M=4$ using symbolic formula manipulation. Smaller approximation orders are not reasonable since they are not superior to the rough finite volume model of Section 2.2 Higher-order approximations are not necessary from a practical point of view since their additional degrees of freedom for the temperature distribution are associated with eigenvalues that are significantly faster than the available Peltier element dynamics.
} 


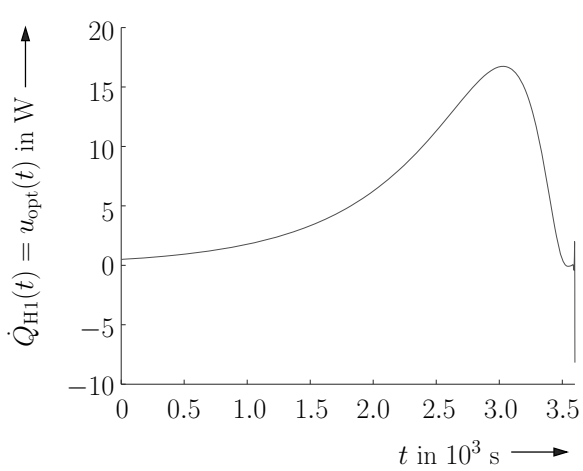

(a)

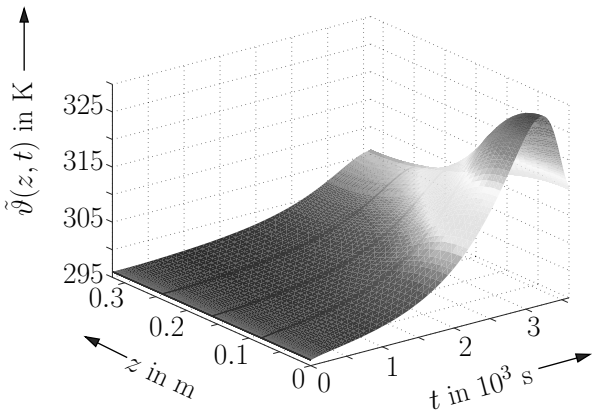

(b)

Fig. 4. Results of energy-optimal control synthesis (optimal feedforward control and reference trajectory): energy-optimal feedforward control strategy $u_{\mathrm{opt}}(\mathrm{a})$, optimized temperature profile for $\dot{Q}_{\mathrm{H} 1}=u_{\mathrm{opt}}$ (b).

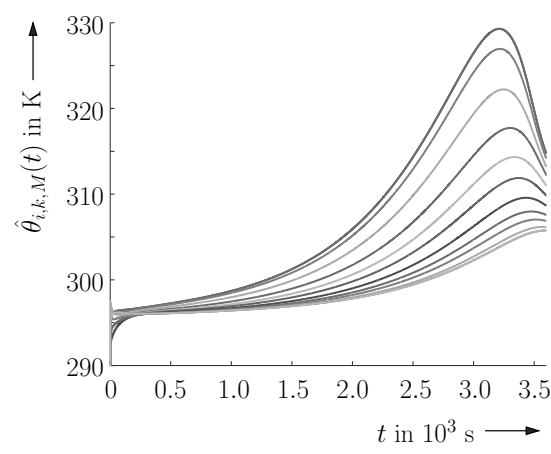

(a)

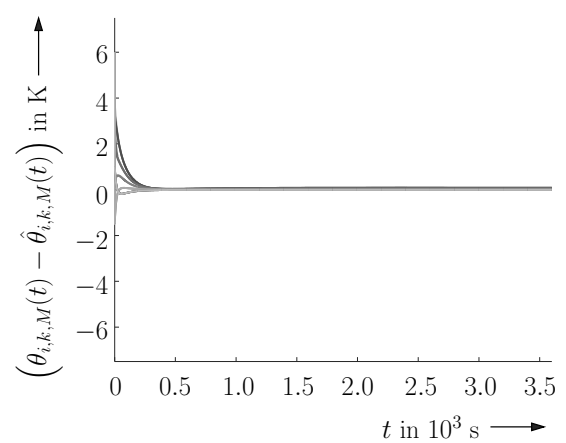

(c)

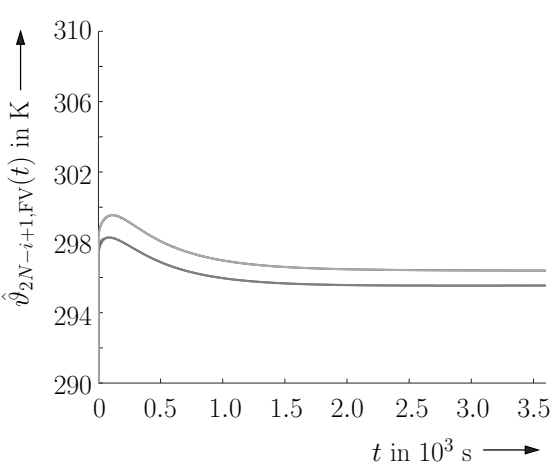

(b)

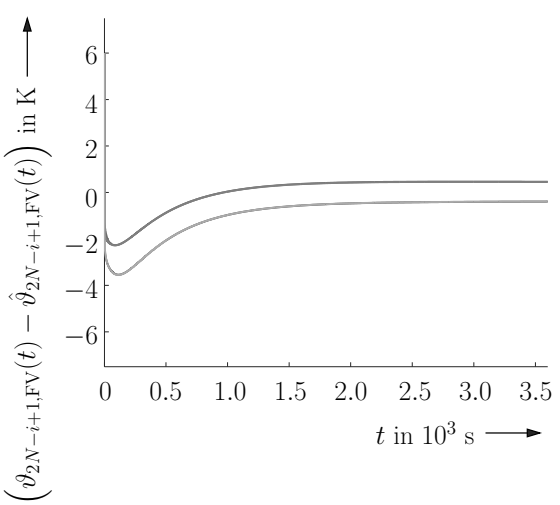

(d)

Fig. 5. Estimation of the coefficients $\Theta_{M}(t)$ and the disturbances $\vartheta_{2 N-i+1, \mathrm{FV}}(t)$ in each rod segment $i \in\{1, \ldots, 4\}$ with initial errors of $6 \mathrm{~K}$ in all variables under consideration of the output definition 442: estimates for the coefficients $\theta_{i, k, M}$ of the Bernstein polynomial-based approximation of the temperature profile in the metallic rod (a), estimates for the disturbances $\vartheta_{2 N-i+1, \mathrm{FV}}\left(\right.$ true values $296 \mathrm{~K}$ ) (b), estimation errors for the coefficients $\theta_{i, k, M}$ (c), estimation errors for $\vartheta_{2 N-i+1, \mathrm{FV}}$ (d).

the rod temperature like an additional convective heat input. Although no direct disturbance compensation (as, e.g., presented by Rauh et al., 2013b) is implemented here, the tracking errors remain in the range $[-0.1,0.1] \mathrm{K}$ (which corresponds to the interval of typical measurement errors of the available PT100 elements in Fig. 1) over the complete time horizon. The resulting control signal is depicted in Fig. 6(b) (right).
After a comparison of the estimation results in Fig. 5 with the results that can be achieved by using temperature measurements in each rod segment (Fig. 7), it can be noticed that the resulting estimation errors and the corresponding transient phases can be reduced further by this extension.

However, the effort for rod temperature measurements becomes twice as large as before. 

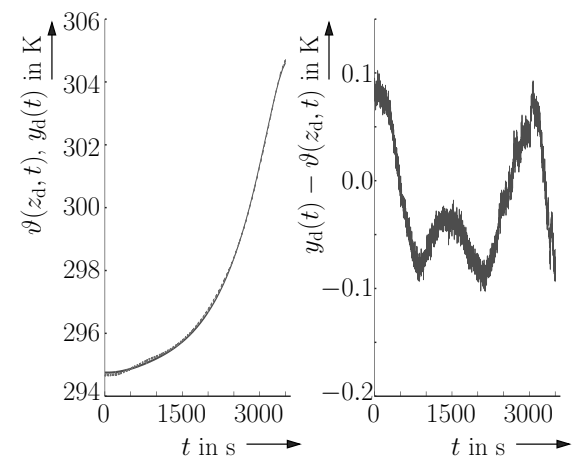

(a)
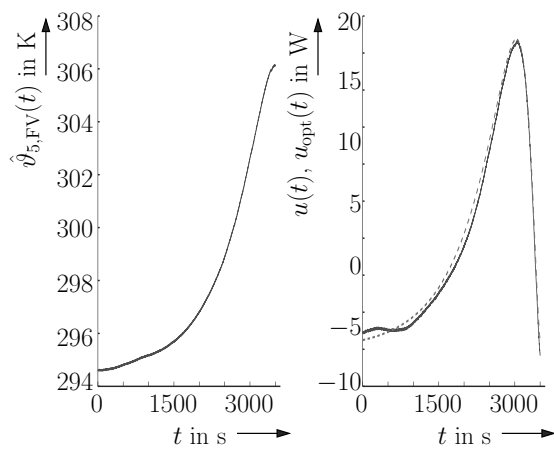

(b)

Fig. 6. Experimental validation of the optimal feedforward control extended by the PI output feedback with the output definition 42): comparison of the desired and actual outputs $y_{\mathrm{d}}(t)$ and $\vartheta\left(z_{\mathrm{d}}, t\right)$ (a), disturbance estimate $\hat{\vartheta}_{5, \mathrm{FV}}(t)$ as well as control $u(t)$ (closed-loop, solid line) and $u_{\mathrm{opt}}(t)$ (offline computed optimal control, dashed line) (b).

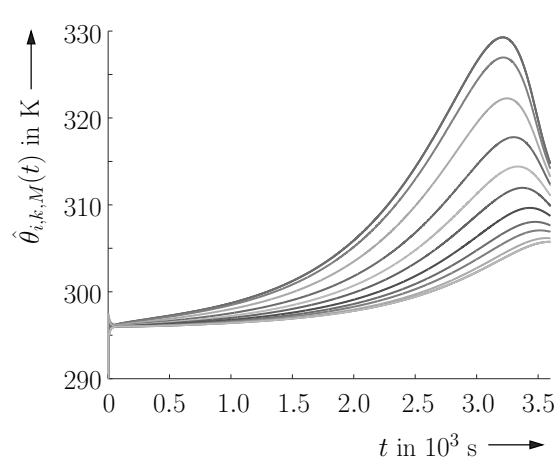

(a)

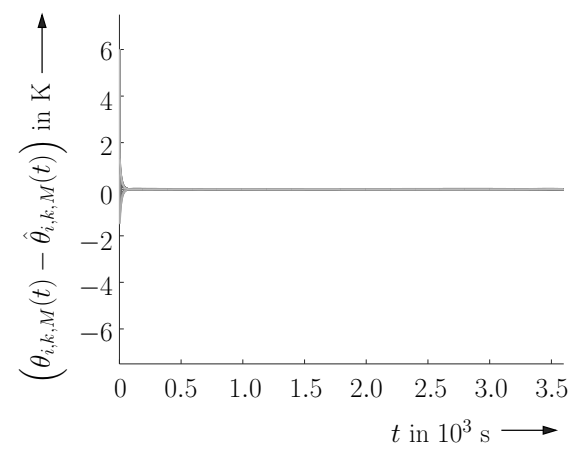

(c)

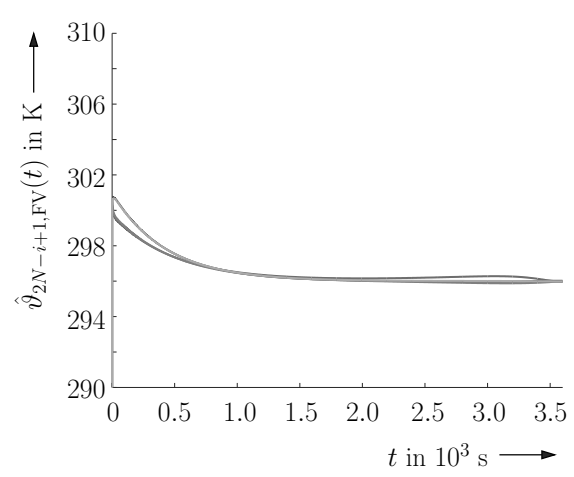

(b)

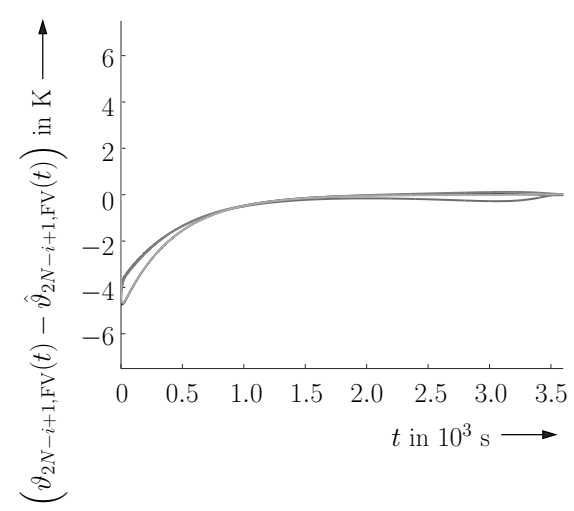

(d)

Fig. 7. Simulation results for the estimation of the coefficients $\boldsymbol{\Theta}_{M}(t)$ and the disturbances $\vartheta_{2 N-i+1, \mathrm{FV}}(t), i \in\{1, \ldots, 4\}$, using the extended output definition (44): estimates for the coefficients $\theta_{i, k, M}$ of the Bernstein polynomial-based approximation of the temperature profile in the metallic rod (a), estimates for the disturbances $\vartheta_{2 N-i+1, \mathrm{FV}}$ (true values $296 \mathrm{~K}$ ) (b), estimation errors for the coefficients $\theta_{i, k, M}$ (c), estimation errors for $\vartheta_{2 N-i+1, \mathrm{FV}}(\mathrm{d})$.

\section{Sensitivity-based predictive control synthesis}

In all simulations and experiments that have been presented so far, it has been assumed that the approxima- ting system model is linear. However, the system has a strong non-linearity at its input if $\dot{m} \neq 0$ holds and if $\dot{m}$ is treated as the control variable.

Hence, the previous linearity assumption is removed in the following while solving the second control task 
defined in Section 2

4.1. Formulation of the sensitivity-based control procedure. To cope with the non-linear behavior, a sensitivity-based predictive control approach is derived in this subsection that consists of a piecewise constant system input $\dot{m}\left(t_{k}\right)$ with the fixed step size $t_{k}-t_{k-1}$. As described by Rauh et al. (2012c), the control is defined by the expression

$$
\dot{m}\left(t_{k}\right)=\dot{m}\left(t_{k-1}\right)+\Delta \dot{m}\left(t_{k}\right)
$$

with

$$
\Delta \dot{m}\left(t_{k}\right)=-\alpha\left(\frac{\partial J_{\mathrm{p}}}{\partial \Delta \dot{m}}\right)^{-1} J_{\mathrm{p}}
$$

and a positive step size control factor $\alpha$. Alternative approaches for a predictive control design can be found in the work of Prodan et al. (2013) and the references therein.

In (47), the variable $J_{\mathrm{p}}$ denotes the value of a performance criterion which is evaluated over the prediction horizon of the length $T_{\mathrm{p}}=N_{\mathrm{p}} \cdot\left(t_{k}-t_{k-1}\right)$ according to

$$
\begin{gathered}
J_{\mathrm{p}}=\sum_{i=k}^{k+N_{\mathrm{p}}} J_{\mathrm{p}, i}, \\
J_{\mathrm{p}, i}= \begin{cases}\left(\vartheta_{\max , i}-w\right)^{2} & \text { for } \vartheta_{\max , i}>w, \\
\left(\dot{m}\left(t_{k-1}\right)+\beta_{\mathrm{p}} \cdot \Delta \dot{m}\left(t_{k}\right)\right)^{2} & \text { otherwise, }\end{cases}
\end{gathered}
$$

where

$$
\vartheta_{\max , i}:=\max _{z \in[0, l]}\left\{\tilde{\vartheta}\left(z, t_{k}+\frac{(i-k) \cdot T_{\mathrm{p}}}{N_{\mathrm{p}}}\right)\right\}
$$

is the predicted maximum rod temperature at the point of time

$$
t_{i}=t_{k}+\frac{(i-k) \cdot T_{\mathrm{p}}}{N_{\mathrm{p}}}
$$

and $\beta_{\mathrm{p}}>0$ is a scaling factor.

The criterion (48) is evaluated online during the state prediction by using an explicit Euler discretization of the ODEs for $\boldsymbol{\Theta}_{M}(t), t \in\left[t_{k}, t_{k+N_{\mathrm{p}}}\right]$, resulting from the MIDR approach with $\dot{m}=\dot{m}\left(t_{k}\right), \Delta \dot{m}\left(t_{k}\right)=0$, and the mass flow-dependent parameters $p_{1}(\dot{m}), \ldots, p_{5}(\dot{m})$, which are given by fixed-order polynomials. During this online evaluation of the state equations for the system model depicted in Fig. 3, the ODEs for $\Theta_{M}$, summarized in the state vector $\boldsymbol{x}$, are coupled with the ODEs for $\vartheta_{5, \mathrm{FV}}, \ldots, \vartheta_{8, \mathrm{FV}}$. The latter ODEs are defined in (2) in such a way that all $\vartheta_{5, \mathrm{FV}}(t) \approx$ $\vartheta_{5, \mathrm{AC}}(z, t), \ldots, \vartheta_{8, \mathrm{FV}}(t) \approx \vartheta_{8, \mathrm{AC}}(z, t)$ are piecewise homogeneous in the expressions for $\dot{\boldsymbol{\Theta}}_{M}$ for each finite element $\left[z_{i-1}, z_{i}\right], i \in\{1, \ldots, 4\}$. Additionally, Eqns. (2) for $\dot{\vartheta}_{5, \mathrm{FV}}, \ldots, \dot{\vartheta}_{8, \mathrm{FV}}$ are evaluated for the temperatures

$$
\vartheta_{i, \mathrm{FV}}(t) \approx \vartheta_{i, \mathrm{FE}}\left(\bar{z}_{i}, t\right), \quad \bar{z}_{i}:=\frac{1}{2}\left(z_{i-1}+z_{i}\right),
$$

at the respective segment midpoints.

During this prediction, the heat flows $\dot{Q}_{\mathrm{H} i}, i \in$ $\{1, \ldots, 4\}$, of the Peltier elements are replaced by estimates that are determined by an extended observer. This observer is similar to the one in the previous section, where $\boldsymbol{\Theta}_{M}\left(t_{k}\right)$ and the air canal temperatures were determined simultaneously; see Section 4.2

In 477, the partial derivative of $J_{\mathrm{p}}$ with respect to a variation in the control input is required. This derivative is determined by means of algorithmic differentiation (Griewank and Walther, 2008) in a $\mathrm{C}++$ implementation of the state equations. For that purpose, the state equations are evaluated after overloading the control increment $\Delta \dot{m}\left(t_{k}\right)$ by the forward differentiation operator that is provided by FADBAD++ (Bendsten and Stauning, 2007). As shown by Rauh et al. (2012a), this procedure can also be generalized to the control of multi-input multi-output systems as well as to state and parameter estimation. Compared with a symbolic computation of the required partial derivatives, algorithmic differentiation leads to implementations with an improved computational efficiency (Röbenack, 2002) and makes the approach also applicable to higher-dimensional non-linear systems with long prediction horizons $N_{\mathrm{p}}$. To make this control approach robust and stable, the discretization step size $t_{k}-t_{k-1}$, the prediction horizon $T_{\mathrm{p}}$, and the parameter $\alpha$ in (47) are chosen thoroughly after simulations of the closed-loop controller under consideration of measurement noise and parameter uncertainty.

4.2. State and disturbance observer design. To make the observer presented in Section 3.3 applicable to the extended non-linear system model, it is assumed that $\vartheta_{6, \mathrm{FV}} \equiv \vartheta_{7, \mathrm{FV}}$ holds. With this assumption, the ODEs obtained from the projection approach of the MIDR are appended by independent integrator disturbance model 6 for the heat flows according to $\frac{\mathrm{d}}{\mathrm{d} t} \dot{Q}_{\mathrm{H} i}=0, i \in$ $\{1, \ldots, 4\}$, and by the disturbance models $\dot{\vartheta}_{5, \mathrm{FV}}=0$, $\dot{\vartheta}_{6, \mathrm{FV}} \equiv \dot{\vartheta}_{7, \mathrm{FV}}=0$, and $\dot{\vartheta}_{8, \mathrm{FV}}=0$ for the air canal temperatures.

It can be shown that this extended system is observable due to the simplifying assumption $\vartheta_{6, \mathrm{FV}} \equiv$ $\vartheta_{7, \mathrm{FV}}$ for each mass flow $\dot{m}$ if at least the rod temperatures $\vartheta_{1, \mathrm{FE}}(l / 8, t)$ and $\vartheta_{4, \mathrm{FE}}(7 l / 8, t)$, the air canal temperatures $\vartheta_{5, \mathrm{FV}}$ and $\vartheta_{8, \mathrm{VE}}$, as well as

\footnotetext{
${ }^{6}$ The integrator disturbance model is reasonable if no a-priori knowledge about the disturbances is available and if the corresponding quantities are constant or slowly varying.
} 
expressions representing the adiabatic insulation of the rod edges according to

$$
\vartheta_{1, \mathrm{FE}}^{\prime}(0, t):=\left.\frac{\partial \vartheta_{1, \mathrm{FE}}(z, t)}{\partial z}\right|_{z=0}=0
$$

and

$$
\vartheta_{4, \mathrm{FE}}^{\prime}(l, t):=\left.\frac{\partial \vartheta_{4, \mathrm{FE}}(z, t)}{\partial z}\right|_{z=l}=0
$$

are used as (virtually) measured data given by

$$
\begin{aligned}
\boldsymbol{y}= & {\left[\vartheta_{1, \mathrm{FE}}\left(\frac{l}{8}, t\right), \vartheta_{4, \mathrm{FE}}\left(\frac{7 l}{8}, t\right), \vartheta_{1, \mathrm{FE}}^{\prime}(0, t), \vartheta_{4, \mathrm{FE}}^{\prime}(l, t),\right.} \\
& \left.\vartheta_{5, \mathrm{FV}}, \vartheta_{8, \mathrm{FV}}, \sum_{i=1}^{4} \dot{Q}_{\mathrm{H} i}\right]^{T}=\boldsymbol{C}_{3}\left[\begin{array}{c}
\boldsymbol{\Theta}_{M} \\
\boldsymbol{z}
\end{array}\right], \\
\boldsymbol{C}:= & \boldsymbol{C}_{3}, \\
\boldsymbol{z}:= & {\left[\vartheta_{5, \mathrm{FV}}, \vartheta_{6, \mathrm{FV}}, \vartheta_{8, \mathrm{FV}}, \dot{Q}_{\mathrm{H} 1}, \dot{Q}_{\mathrm{H} 2}, \dot{Q}_{\mathrm{H} 3}, \dot{Q}_{\mathrm{H} 4}\right]^{T} }
\end{aligned}
$$

with

$$
\boldsymbol{C}_{3}=\left[\begin{array}{cccccc}
\boldsymbol{b}_{1, M}^{T}\left(\frac{l}{8}\right) & \mathbf{0}_{M+1}^{T} & \mathbf{0}_{M+1}^{T} & \mathbf{0}_{M+1}^{T} & \mathbf{0}_{3}^{T} & \mathbf{0}_{4}^{T} \\
\mathbf{0}_{M+1}^{T} & \mathbf{0}_{M+1}^{T} & \mathbf{0}_{M+1}^{T} & \boldsymbol{b}_{4, M}^{T}\left(\frac{7 l}{8}\right) & \mathbf{0}_{3}^{T} & \mathbf{0}_{4}^{T} \\
\boldsymbol{b}_{1, M}^{T}(0) & \mathbf{0}_{M+1}^{T} & \mathbf{0}_{M+1}^{T} & \mathbf{0}_{M+1}^{T} & \mathbf{0}_{3}^{T} & \mathbf{0}_{4}^{T} \\
\mathbf{0}_{M+1}^{T} & \mathbf{0}_{M+1}^{T} & \mathbf{0}_{M+1}^{T} & \boldsymbol{b}_{4, M}^{T}(l) & \mathbf{0}_{3}^{T} & \mathbf{0}_{4}^{T} \\
\mathbf{0}_{M+1}^{T} & \mathbf{0}_{M+1}^{T} & \mathbf{0}_{M+1}^{T} & \mathbf{0}_{M+1}^{T} & {[100]} & \mathbf{0}_{4}^{T} \\
\mathbf{0}_{M+1}^{T} & \mathbf{0}_{M+1}^{T} & \mathbf{0}_{M+1}^{T} & \mathbf{0}_{M+1}^{T} & {[001]} & \mathbf{0}_{4}^{T} \\
\mathbf{0}_{M+1}^{T} & \mathbf{0}_{M+1}^{T} & \mathbf{0}_{M+1}^{T} & \mathbf{0}_{M+1}^{T} & \mathbf{0}_{3}^{T} & \mathbf{1}_{4}^{T}
\end{array}\right],
$$

$$
\mathbf{1}_{4}:=\left[\begin{array}{llll}
1 & 1 & 1 & 1
\end{array}\right]^{T} .
$$

In (51), it is necessary to include the sum of all heat flows $\sum_{i=1}^{4} \dot{Q}_{\mathrm{H} i}$ without, however, any knowledge about the spatial distribution 7 To improve the robustness of the observer, further temperature measurements $\vartheta_{2, \mathrm{FE}}(3 l / 8, t)$ and $\vartheta_{3, \mathrm{FE}}(5 l / 8, t)$ could be included as before in the combined state and disturbance observer

$$
\dot{\tilde{\tilde{x}}}(t)=\hat{\boldsymbol{f}}(\hat{\tilde{\boldsymbol{x}}}(t), \dot{m}(t))+\boldsymbol{L}(\boldsymbol{y}(t)-\hat{\boldsymbol{y}}(t))
$$

with

$$
\begin{aligned}
\hat{\boldsymbol{f}}(\hat{\tilde{\boldsymbol{x}}}, \dot{m}) \\
:=\left[\left(\boldsymbol{A}(\dot{m}) \hat{\boldsymbol{x}}+\boldsymbol{B}\left[\begin{array}{c}
\hat{\dot{Q}}_{\mathrm{H} 1} \\
\dot{\hat{Q}}_{\mathrm{H} 2} \\
\hat{\dot{Q}}_{\mathrm{H} 3} \\
\hat{\dot{Q}}_{\mathrm{H} 4}
\end{array}\right]+\boldsymbol{E}(\dot{m})\left[\begin{array}{c}
\vartheta_{5, \mathrm{FV}} \\
\vartheta_{6, \mathrm{FV}} \\
\vartheta_{6, \mathrm{FV}} \\
\vartheta_{8, \mathrm{FV}}
\end{array}\right]\right)^{T} \mathbf{0}_{7}^{T}\right]^{T} .
\end{aligned}
$$

\footnotetext{
${ }^{7}$ This heat flow measurement as well as the information about two air canal temperatures is necessary to distinguish between changes in the air canal temperature and variations in the Peltier element heat flows in a model-based way. If fewer measurements were available, only a joint heat flow to the ambience could be estimated for each of the rod segments. A distinction between heat convection between the rod and the air canal and the Peltier element heat flows would become impossible without measuring the sum of all Peltier element heat flows.
}

Here, the rod temperature is described by the finite element version of the MIDR and the air canal by the finite volume model. As before, the gain matrix $\boldsymbol{L}=\boldsymbol{L}(\dot{m})$ is calculated by minimizing an error measure which is defined in analogy to Eqn. (39). The computation of the observer gain $\boldsymbol{L}(\dot{m})$ has been performed offline for ten equally spaced grid points covering the complete range of the air mass flow $\dot{m}$. During the online evaluation of the observer, the corresponding gain values are interpolated linearly at each point of time $t_{k}$ by using the actual control signal $\dot{m}\left(t_{k}\right)$.

\subsection{Experimental results for sensitivity-based pre-} dictive control. In this subsection, experimental results are presented for the control of the heating system by means of variations of the mass flow in the air canal. The prerequisite for its implementation is the information about the spatial distribution of the heat flows $\dot{Q}_{\mathrm{H} i}$ and the online reconstruction of the rod temperature at all points of time. The observer introduced in Section 4.2 is a promising approach to solve these tasks. Furthermore, it helps one to detect the generally time-varying rod position $z^{*}(t)$ at which the maximum temperature can be expected. For that purpose, the coefficients of the temperature distribution are reconstructed first. Then, the first-order derivative of $\tilde{\vartheta}(z, t)$ with respect to the position coordinate $z$ is determined and afterwards set to zero. The corresponding positions as well as the edges of each rod segment are candidates for the location with the maximum temperature. Alternatively, a conservative bound for the maximum rod temperature can be determined as $\max \left\{\boldsymbol{\Theta}_{M}(t)\right\}$ and used by the predictive controller.

Figure 8 visualizes that the predictive control procedure leads to rod temperatures which do not show any noticeable overshoot over the time-varying limit value $w(t)$ if $T_{\mathrm{p}}=8 \mathrm{~s}, N_{\mathrm{p}}=40, \alpha=10^{-3}, \beta_{\mathrm{p}}=0.1$ and a control sampling time of $1 \mathrm{~s}$ are used. Moreover, a safety bound of $0.6 \mathrm{~K}$ has been added to the temperatures that are estimated for each of the rod segments by means of the state observer. This safety bound accounts for estimation errors during the swing-in phase for the heat flows $\dot{Q}_{\mathrm{H} i}$. The value of this safety bound has been determined from an offline simulation to account for state reconstruction errors during transient phases. The current work aims at extensions of the presented controller towards a learning-type approach that can be used for tracking temperature profiles which are periodic with respect to time.

\section{Conclusions and outlook on future work}

In this paper, modeling procedures and control algorithms with real-time capable state and disturbance observers have been derived for both robust trajectory tracking and optimal control of distributed heating systems. These 

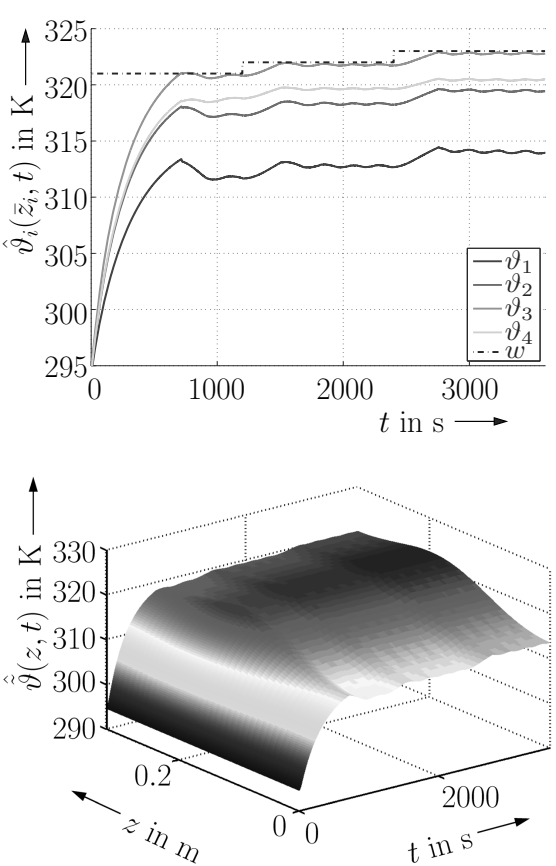
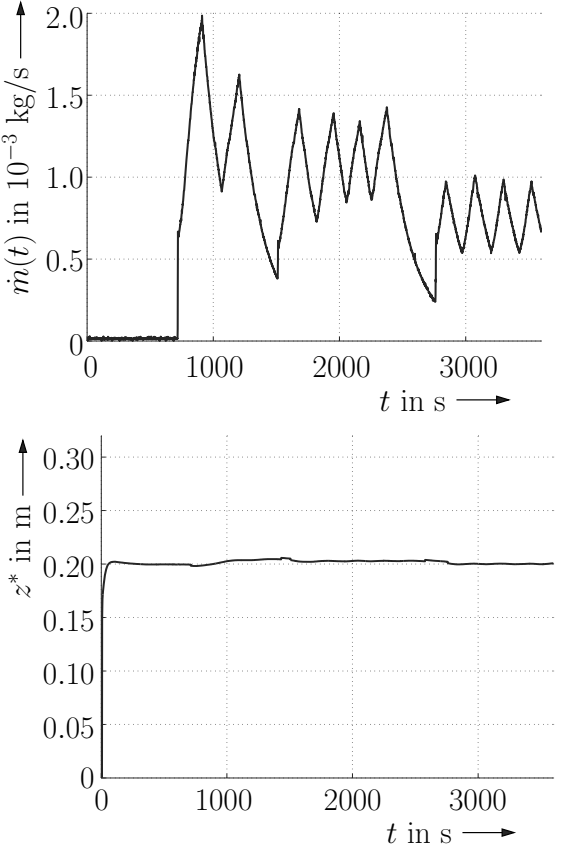

Fig. 8. Measured temperatures and control input for the predictive control (top), estimates for $\vartheta(z, t)$ and the location $z^{*}$ of the maximum rod temperature (bottom).

approaches have been extended by a sensitivity-based predictive controller which allows temperature control of a metallic rod despite non-linearities caused by an adjustable air stream on its top.

All control strategies are based on the MIDR, a projection approach, and a novel control-oriented finite element technique. This finite element approach makes use of a parameterization of the temperature distribution on the basis of Bernstein polynomials. This type of approximation simplifies the definition of boundary and inter-element conditions as well as the computation of worst-case bounds of the temperature profile in both space and time. Furthermore, it can be generalized in a straightforward manner to spatially higher-dimensional problems (see Rauh et al., 2013b; Kersten et al., 2014).

The experimental validation of all presented controllers has shown accurate trajectory tracking as well as the capability of reliable estimation of non-measurable system states and disturbances. Moreover, the MIDR provides explicit estimates for both the local and integral quality of the mathematical description of the temperature distribution. These estimates help us to systematically improve the approximation quality by adapting the number of finite elements and the polynomial orders.

In future work, the presented control procedures will be validated further in experiments for suitable test rigs of the above-mentioned higher-dimensional heating systems that are available at the Chair of Mechatronics at the University of Rostock. There, order reduction techniques such as the ones mentioned by Janiszowski (2014) may become relevant to guarantee real-time applicability of control and state estimation procedures.

\section{Acknowledgment}

This work was supported by the Russian Foundation for Basic Research, projects no. 12-01-00789, 13-01-00108, 14-01-00282, the Leading Scientific Schools Grants NSh-2710.2014.1, NSh-2954.2014.1, and by the German Research Foundation (Deutsche Forschungsgemeinschaft DFG) under the grant no. AS 132/2-1.

\section{References}

Aschemann, H., Kostin, G.V., Rauh, A. and Saurin, V.V. (2010). Approaches to control design and optimization in heat transfer problems, Journal of Computer and Systems Sciences International 49(3): 380-391.

Åström, K. (1970). Introduction to Stochastic Control Theory, Mathematics in Science and Engineering, Academic Press, New York, NY/London.

Bachmayer, M., Ulbrich, H. and Rudolph, J. (2011). Flatness-based control of a horizontally moving erected beam with a point mass, Mathematical and Computer Modelling of Dynamical Systems 17(1): 49-69.

Bendsten, C. and Stauning, O. (2007). FADBAD++, Version 2.1, http: //www. fadbad.com

Deutscher, J. and Harkort, C. (2008). Vollständige Modale Synthese eines Wärmeleiters, Automatisierungstechnik 56(10): 539-548. 
Deutscher, J. and Harkort, C. (2010). Entwurf endlich-dimensionaler Regler für lineare verteilt parametrische Systeme durch Ausgangsbeobachtung, Automatisierungstechnik 58(8): 435-446.

Gehring, N., Knüppel, T., Rudolph, J. and Woittennek, F. (2012). Parameter identification for a heavy rope with internal damping, Proceedings in Applied Mathematics and Mechanics 12(1): 725-726.

Griewank, A. and Walther, A. (2008). Evaluating Derivatives: Principles and Techniques of Algorithmic Differentiation, SIAM, Philadelphia, PA.

Janiszowski, K.B. (2014). Approximation of a linear dynamic process model using the frequency approach and a non-quadratic measure of the model error, International Journal of Applied Mathematics and Computer Science 24(1): 99-109, DOI: 10.2478/amcs-2014-0008.

Kersten, J., Rauh, A. and Aschemann, H. (2014). Finite element modeling for heat transfer processes using the method of integro-differential relations with applications in control engineering, IEEE International Conference on Methods and Models in Automation and Robotics MMAR 2014, Międzyzdroje, Poland, pp. 606-611.

Kharitonov, A. and Sawodny, O. (2006). Flatness-based disturbance decoupling for heat and mass transfer processes with distributed control, IEEE International Conference on Control Applications CCA, Munich, Germany, pp. 674-679.

Kostin, G. and Saurin, V. (2012). Integrodifferential Relations in Linear Elasticity, De Gruyter, Berlin.

Kostin, G.V. and Saurin, V.V. (2006). Modeling of controlled motions of an elastic rod by the method of integro-differential relations, Journal of Computer and Systems Sciences International 45(1): 56-63.

Krstic, M. and Smyshlyaev, A. (2010). Adaptive Control of Parabolic PDEs, Princeton University Press, Princeton, NJ.

Lions, J.L. (1971). Optimal Control of Systems Governed by Partial Differential Equations, Springer-Verlag, New York, NY.

Malchow, F. and Sawodny, O. (2011). Feedforward control of inhomogeneous linear first order distributed parameter systems, American Control Conference on ACC 2011, San Francisco, CA, USA, pp. 3597-3602.

Meurer, T. and Kugi, A. (2009). Tracking control for boundary controlled parabolic PDEs with varying parameters: Combining backstepping and differential flatness, Automatica 45(5): 1182-1194.

Meurer, T. and Zeitz, M. (2003). A novel design of flatness-based feedback boundary control of nonlinear reaction-diffusion systems with distributed parameters, in W. Kang, M. Xiao and C. Borges (Eds.), New Trends in Nonlinear Dynamics and Control, Lecture Notes in Control and Information Science, Vol. 295, Springer, Berlin/Heidelberg, pp. 221-236.

Prodan, I., Olaru, S., Stoica, C. and Niculescu, S.-I. (2013). Predictive control for trajectory tracking and decentralized navigation of multi-agent formations, International Journal of Applied Mathematics and Computer Science 23(1): 91-102, DOI: 10.2478/amcs-2013-0008.

Rauh, A. and Aschemann, H. (2012). Parameter identification and observer-based control for distributed heating systems - the basis for temperature control of solid oxide fuel cell stacks, Mathematical and Computer Modelling of Dynamical Systems 18(4): 329-353.

Rauh, A., Butt, S.S. and Aschemann, H. (2013a). Nonlinear state observers and extended Kalman filters for battery systems, International Journal of Applied Mathematics and Computer Science 23(3): 539-556, DOI: 10.2478/amcs-2013-0041.

Rauh, A., Dittrich, C. and Aschemann, H. (2013b). The method of integro-differential relations for control of spatially two-dimensional heat transfer processes, European Control Conference ECC'13, Zurich, Switzerland, pp. 572-577.

Rauh, A., Kostin, G.V., Aschemann, H., Saurin, V.V. and Naumov, V. (2010). Verification and experimental validation of flatness-based control for distributed heating systems, International Review of Mechanical Engineering 4(2): $188-200$.

Rauh, A., Senkel, L. and Aschemann, H. (2012a). Sensitivity-based state and parameter estimation for fuel cell systems, 7th IFAC Symposium on Robust Control Design, Aalborg, Denmark, pp. 57-62.

Rauh, A., Senkel, L., Aschemann, H., Kostin, G.V. and Saurin, V.V. (2012b). Reliable finite-dimensional control procedures for distributed parameter systems with guaranteed approximation quality, IEEE Multi-Conference on Systems and Control, Dubrovnik, Croatia, pp. 214-219.

Rauh, A., Senkel, L., Dittrich, C. and Aschemann, H. (2012c). Observer-based predictive temperature control for distributed heating systems based on the method of integrodifferential relations, IEEE International Conference on Methods and Models in Automation and Robotics MMAR 2012, Międzyzdroje, Poland, pp. 528-533.

Rauh, A., Warncke, J., Kostin, G.V., Saurin, V.V. and Aschemann, H. (2015). Numerical validation of order reduction techniques for finite element modeling of a flexible rack feeder system, European Control Conference ECC'15, Linz, Austria, pp. 1094-1099.

Röbenack, K. (2002). On the efficient computation of higher order maps $a d_{f}^{k} g(x)$ using Taylor arithmetic and the Campbell-Baker-Hausdorff formula, in A. Zinober and D. Owens (Eds.), Nonlinear and Adaptive Control, Lecture Notes in Control and Information Science, Vol. 281, Springer, Berlin/Heidelberg, pp. 327-336.

Saurin, V.V., Kostin, G.V., Rauh, A. and Aschemann, H. (2011a). Adaptive control strategies in heat transfer problems with parameter uncertainties based on a projective approach, in A. Rauh and E. Auer (Eds.), Modeling, Design, and Simulation of Systems with Uncertainties, Mathematical Engineering, Springer, Berlin/Heidelberg, pp. 309-332. 
Saurin, V.V., Kostin, G.V., Rauh, A. and Aschemann, H. (2011b). Variational approach to adaptive control design for distributed heating systems under disturbances, International Review of Mechanical Engineering 5(2): 244-256.

Saurin, V.V., Kostin, G.V., Rauh, A., Senkel, L. and Aschemann, H. (2012). An integrodifferential approach to adaptive control design for heat transfer systems with uncertainties, 7th Vienna International Conference on Mathematical Modelling MATHMOD 2012, Vienna, Austria, pp. 520-525.

Stengel, R. (1994). Optimal Control and Estimation, Dover Publications, Inc., Mineola, NY.

Tao, G. (2003). Adaptive Control Design and Analysis, Wiley \& Sons, Inc., Hoboken, NJ.

Thull, D., Kugi, A. and Schlacher, K. (2010). Tracking Control of Mechanical Distributed Parameter Systems with Applications, Shaker-Verlag, Aachen.

Touré, Y. and Rudolph, J. (2002). Controller design for distributed parameter systems, Encyclopedia of Life Support Systems (EOLSS), http://www.eolss.net/sample-chapters / c18/E6-43-19-02.pdf

Utz, T., Meurer, T. and Kugi, A. (2011). Trajectory planning for a two-dimensional quasi-linear parabolic PDE based on finite difference semi-discretizations, 18th IFAC World Congress, Milano, Italy, pp. 12632-12637.

Winkler, F.J. and Lohmann, B. (2009). Flatness-based control of a continuous furnace, 3rd IEEE Multi-Conference on Systems and Control (MSC), Saint Petersburg, Russia, pp. 719-724.

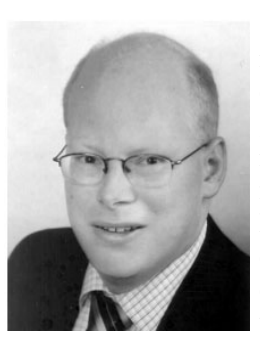

Andreas Rauh was born in Munich, Germany, in 1977. He received his diploma degree in electrical engineering and information technology from Technische Universität München, Munich, in 2001, and his Ph.D. degree (Dr.-Ing.) from the University of Ulm, Germany, in 2008. He has published more than 130 articles and chapters in edited books, international conferences and peer-reviewed journals. His research interests are state and parameter estimation for stochastic and set-valued uncertainties, verified simulation of nonlinear uncertain systems, non-linear, robust, and optimal control, interval methods for ordinary differential equations as well as differential-algebraic systems. Dr. Rauh is currently with the Chair of Mechatronics, University of Rostock, Germany, as a post-doctoral research associate. Since 2008 he has been a member of the IEEE 1788 Working Group for the Standardization of Interval Arithmetic. Moreover, in 2011, he was elected a corresponding member of the International Academy of Engineering, Moscow, Russia.

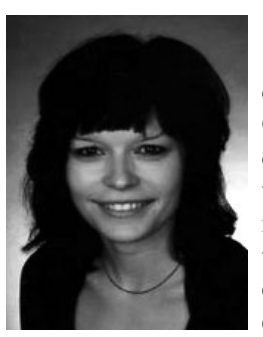

Luise Senkel was born in Görlitz, Germany, 1989. She received her M.Sc. degree in mechanical engineering from the University of Rostock, Germany, in 2012. Currently, she is a Ph.D. student at the Chair of Mechatronics at the University of Rostock, and works on the development of interval methods for robust control and estimation of uncertain dynamic systems as well as on control and observer synthesis for solid oxide fuel cells and distributed parameter systems. Luise
Senkel is an author/co-author of more than 30 articles in international conferences and peer-reviewed journals.

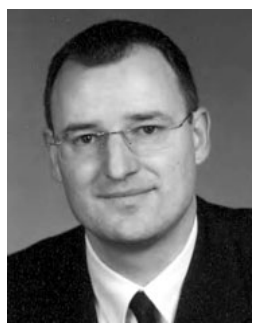

Harald Aschemann was born in Hildesheim, Germany, in 1966. He received his diploma degree in mechanical engineering from the University of Hanover, Germany, in 1994. After two years of engagement in research and development with a leading company in machine tools, where he worked on automated transfer systems, he joined the Department of Measurement, Control, and Microtechnology at the University of Ulm, Germany. He completed his Ph.D. (Dr.Ing.) on optimal trajectory planning and trajectory control of an overhead traveling crane in 2001. From 2001 till 2006, he proceeded as a research associate and lecturer at the same department. Since 2006, Harald Aschemann has been a full professor and the head of the Chair of Mechatronics at the University of Rostock, Germany. His research interests involve control-oriented modeling, identification, non-linear control, and simulation of mechatronic, robotic and thermofluidic systems. In 2011, he was elected a corresponding member of the International Academy of Engineering, Moscow, Russia.

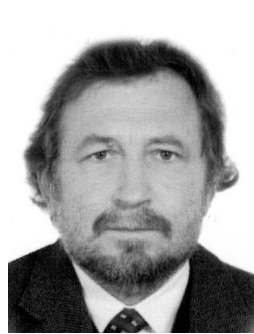

Vasily V. Saurin was born in the Rostov region, Russia, in 1961. In 1984, he received his Master's degree from the Moscow Institute of Physics and Technology (MIPT), Russia, in 1992 the Ph.D. degree from the Kazan Aviational Institute, Kazan, Russia, and in 2014 the Dr. Sci. degree from the RAS Institute for Problems in Mechanics (IPM), Moscow. He has published a monograph as well as more than 140 articles in conference proceedings, edited books, and peerreviewed journals. His research interests focus on mechanics, mechatronics, and optimal control. Dr. Saurin is currently a senior researcher at the IPM and a member of EUROMECH (European Mechanics Society).

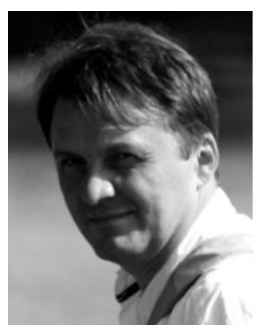

Georgy V. Kostin was born in the Rostov region, Russia, in 1965. In 1982 he received his Master's degree from the Moscow Institute of Physics and Technology (MIPT), Russia, in 1992 the Ph.D. degree from the Institute of Applied Mathematics of the Russian Academy of Sciences (RAS), Moscow, and in 2014 the Dr.Sci. degree from the RAS Institute for Problems in Mechanics (IPM), Moscow. He has published a monograph as well as more than 120 articles in conference proceedings, edited books, and peer-reviewed journals. His research interests focus on mechanics, mechatronics, and optimal control. Dr. Kostin is currently a senior researcher at the IPM and the vice-head of the Chair of Mechanics and Control Processes at the MIPT. He is a fellow of AvH (Alexander von Humboldt Foundation) and a member of GAMM (Gesellschaft für Angewandte Mathematik und Mechanik) as well as EUROMECH (European Mechanics Society).

Received: 19 September 2014 Revised: 28 May 2015

Re-revised: 12 September 2015 